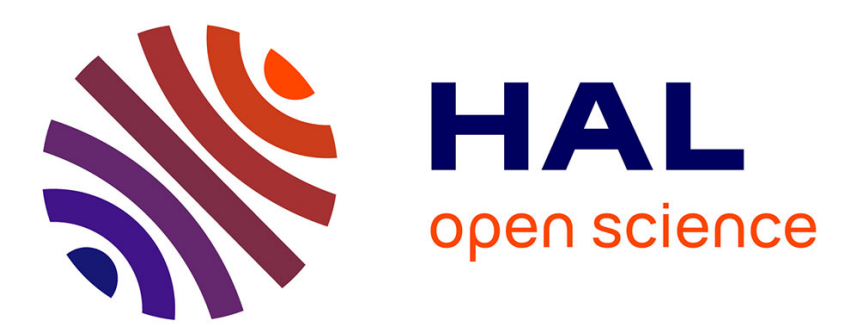

\title{
Morphologie gabaritique et apophonie dans un langage secret féminin (taqjmit) en berbère tachelhit
}

\author{
Mohamed Lahrouchi, Philippe Ségéral
}

\section{To cite this version:}

Mohamed Lahrouchi, Philippe Ségéral. Morphologie gabaritique et apophonie dans un langage secret féminin (taqjmit) en berbère tachelhit. Canadian Journal of Linguistics / Revue canadienne de linguistique, 2009, 54 (2), pp.291-316. 10.1017/S0008413100001262 . hal-00437910

\section{HAL Id: hal-00437910 https://hal.science/hal-00437910}

Submitted on 15 Jun 2021

HAL is a multi-disciplinary open access archive for the deposit and dissemination of scientific research documents, whether they are published or not. The documents may come from teaching and research institutions in France or abroad, or from public or private research centers.
L'archive ouverte pluridisciplinaire HAL, est destinée au dépôt et à la diffusion de documents scientifiques de niveau recherche, publiés ou non, émanant des établissements d'enseignement et de recherche français ou étrangers, des laboratoires publics ou privés. 


\section{Morphologie gabaritique et apophonie \\ dans un langage secret féminin (taqjmit) \\ en berbère tachelhit}

Mohamed Lahrouchi \& Philippe Ségéral

\section{Introduction}

Les langages secrets et les jeux de langage ont fait l'objet, dans les dernières décennies, de nombreuses études qui ont contribué de manière significative au progrès des théories morphophonologiques ${ }^{1}$. Ils ont servi à démontrer, de manière claire, que les locuteurs qui les pratiquent ont accès à des niveaux de représentation abstraits de leur langue. Le présent article s'inscrit dans cette tradition.

Nous considérerons dans ce papier un taqjmit. Sous ce terme ou celui de ta Sjmit (de l'arabe al Sajamia "langue étrangère, incompréhensible"), on désigne habituellement les langages secrets du berbère.

La variété de taqjmit qui sera étudiée ici (et que nous désignerons dans la suite comme le taqjmit) est celle pratiquée en tachelhit par des femmes d'Issouktane, un village situé près de Taliouine, à une centaine de kilomètres au sud-est d'Agadir. Les données ont été collectées par l'un des co-auteurs en 1994 auprès d'une informatrice âgée de 20 ans et ont été complétées par lui en 2006 auprès d'une octogénaire, toutes deux originaires d'Issouktane et toutes deux monolingues - la première ayant néanmoins une connaissance seconde de l'arabe marocain.

Notre objectif est de montrer que :

1. la compréhension des principes de déguisement des formes dans ce langage secret suppose crucialement l'existence d'un gabarit construit, au sens de celui proposé par Guerssel \& Lowenstamm 1993 pour l'arabe classique, c'est-à-

\footnotetext{
${ }^{1}$ Voir - entre autres : Bagemihl 1987, 1988, 1989, 1991; Hombert 1973, 1986 ; McCarthy 1979, 1981, 1985, 1991 ; Plénat 1984, 1985. Pour une synthèse sur les apports de l'étude des jeux de langage à la théorie phonologique, voir Bagemihl 1991.
} 
dire incluant un site morphologique, de forme $\mathrm{CV}$, qui détermine le type d'opérations morphophonologiques menant à la forme déguisée ;

2. la mélodie vocalique des formes en taqjmit est une instantiation du mécanisme apophonique mis en évidence pour l'arabe classique par Guerssel \& Lowenstamm 1993 et 1996 et généralisé par Ségéral 1995.

L'article s'articule en six sections : dans la section 1, nous examinons les données du taqjmit ; nous introduisons dans la section 2 les points théoriques qui sous-tendent notre approche; ensuite, l'analyse elle-même est menée en trois étapes successives : l'analyse des formations triconsonantiques qui conduit à proposer le gabarit fondamental du taqjmit, puis l'analyse de la mélodie vocalique, enfin l'analyse des formations bi- et monoconsonantiques (sections 3 à 5); nous récapitulons nos conclusions dans la section 6 .

\section{Le taqjmit : examen des données}

Les données du taqjmit sont présentées en [1] :

\begin{tabular}{|c|c|}
\hline tachelhit & taqjmit \\
\hline frh & tiffarhjurh \\
\hline krf & tikkarfjurf \\
\hline $\mathrm{k} \int \mathrm{m}$ & tikka $\int m j u \int m$ \\
\hline $\mathrm{krz}$ & tikkarzjurz \\
\hline sgl & tssagljugl \\
\hline isliw & tissalwjulw \\
\hline $\mathrm{iksud}^{\mathrm{f}}$ & tikkasd $^{\mathrm{f}}$ jusd $^{\mathrm{S}}$ \\
\hline smun & tissamnjumn \\
\hline sawl & tissawljuwl \\
\hline izwir & tizzawrjuwr \\
\hline adyar & tiddayrjuyr \\
\hline $\operatorname{argaz}$ & tirragzjugz \\
\hline tamyart & timmayrjuyr \\
\hline ssry & tissaryjury \\
\hline wwarg & tiwwargjurg \\
\hline ggawr & tiggawrjuwr \\
\hline ssknu & tissaknjukn \\
\hline nqqb & tinnaqbjuqb \\
\hline summr & tissamrjumr \\
\hline 3gugl & tiz3agljugl \\
\hline laxbar & tixxabrjubr \\
\hline
\end{tabular}

sois heureux!
attache!
entre!
laboure!
couvre! enterre!
sois mou!
aie peur!
ramasse!
parle!
devance!
morceau de peau
homme
femme
allume!
rêve!
assieds-toi!
fais pencher!
troue!
bronze au soleil!
suspends-toi!
informations




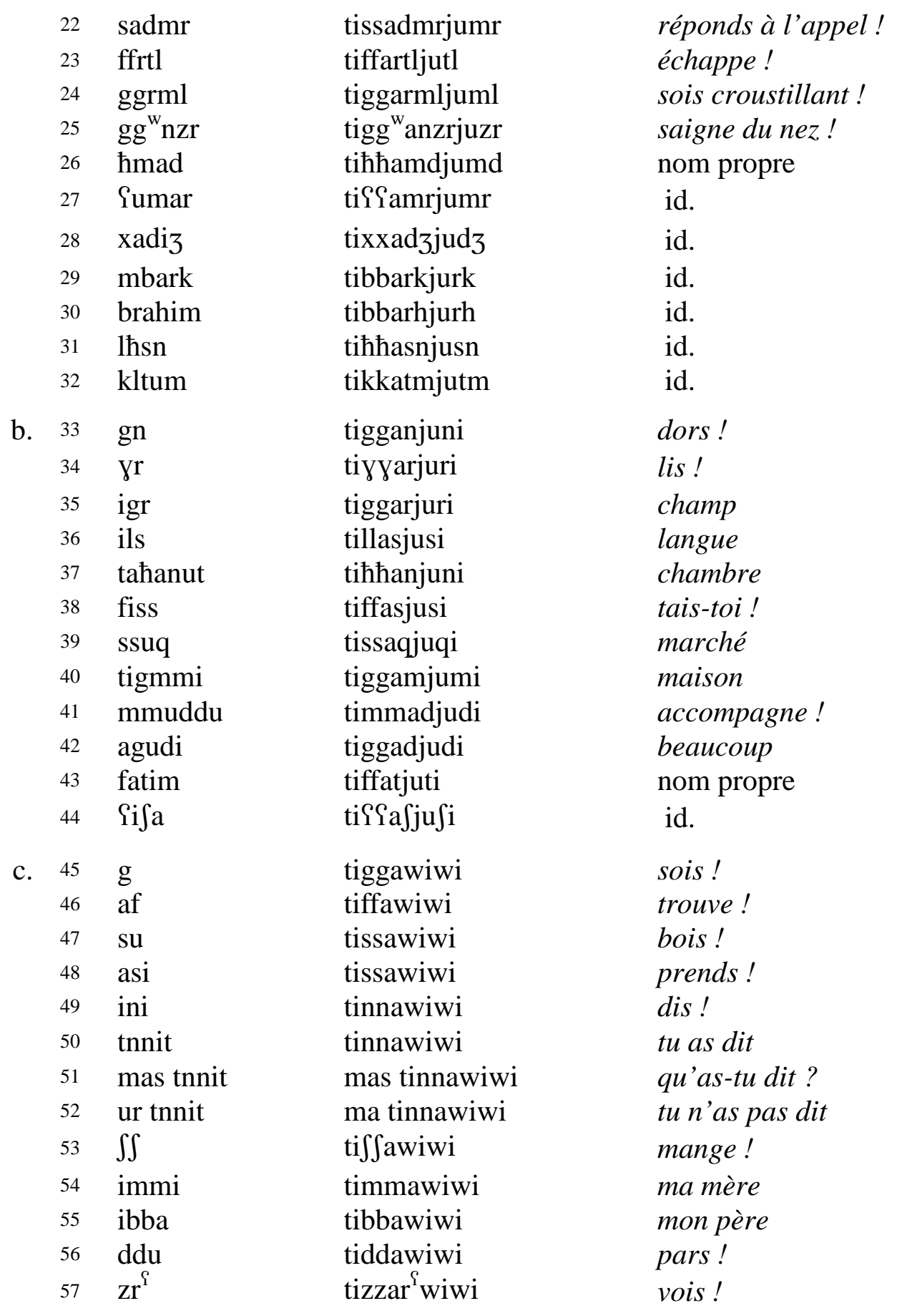

Comme il apparaît, nous avons regroupé les données en [1] en trois classes $\mathrm{a}, \mathrm{b}$ et $\mathrm{c}$. La totalité des formes ${ }^{2} \mathrm{du}$ taqjmit se ramènent en effet à trois schèmes de surface seulement ${ }^{3}$, listés ci-dessous en [2] :

\footnotetext{
${ }^{2}$ Les particules négative et interrogative échappent à cette généralisation. La particule de négation ur est remplacée par ma (qui correspond probablement au morphème de négation de l'arabe
} 
[2]
a.
t i $\mathbf{C}_{1} \mathbf{C}_{1}$ a $\mathbf{C}_{2} \mathbf{C}_{3} \mathbf{j}$ u $\mathbf{C}_{2} \mathbf{C}_{3}=[1 \mathrm{a}]$
b.
t i $\mathrm{C}_{1} \mathrm{C}_{1}$ a $\mathrm{C}_{2}$ j u $\mathrm{C}_{2}$ i
$=[1 \mathrm{~b}]$
c.
t i $\mathbf{C}_{1} \mathbf{C}_{1}$ a w i w i
$=[1 \mathrm{c}]$

Une mélodie vocalique particulière, indépendante de celle de la forme tachelhit correspondante, caractérise chacun de ces schèmes :

$$
i-a\left\{\begin{array}{l}
-u \\
-u-i \\
-i-i
\end{array}\right\} \begin{aligned}
& {[2 a]} \\
& {[2 b]} \\
& {[2 c]}
\end{aligned}
$$

Ces mélodies différentes commencent toutefois toutes les trois par la même partie commune i - a. Et dans la partie initiale des formes, d'ailleurs, ce n'est pas seulement la mélodie qui est commune mais également le matériel consonantique : dans tous les cas, une forme taqjmit commence par $\mathrm{tiC}_{1} \mathrm{C}_{1} \mathrm{a}$-. En termes de morphologie prosodique, ceci pourrait s'interpréter comme un cas de "prosodic circumscription" (McCarthy \& Prince 1990) : la partie invariante des formes taqjmit correspondrait à la portion "prosodiquement circonscrite". Elle est comparable en effet à ce que les deux auteurs cités observent dans les pluriels brisés de l'arabe classique rimaal sables, nufuus âmes, zazaa?ir îles, zawaamiis buffles etc. qui présentent tous une séquence initiale invariante, "prosodiquement circonscrite", de forme $\mathrm{C}_{1} \mathrm{VC}_{2} \mathrm{VV}$-. Cette interprétation possible sera discutée à la fin de cette section lors de l'étude de la partie variable (droite).

Les éléments consonantiques notés "C" en [2], quant à eux, sont les seules informations segmentales communes à la forme en tachelhit et à la forme en taqjmit.

On notera tout d'abord à leur propos qu'une géminée présente dans le tachelhit n'implique pas nécessairement une géminée dans la forme déguisée : fiss $\rightarrow$ tiffasjusi $\left(\mathrm{n}^{\circ} 38\right)$, tigmmi $\rightarrow$ tiggamjumi $\left(\mathrm{n}^{\circ} 40\right)$. La réciproque est également

marocain) : ur tnnit $\rightarrow$ ma tinnawiwi). La particule interrogative mas, quant à elle, est maintenue sans changement.

${ }^{3}$ Pour les cas de sadmr, ffrtl, ggrml, gg ${ }^{\mathrm{w}}$ nzr (respectivement $\mathrm{n}^{\text {os }} 22,23,24$ et 25 en [1]), voir p. 8. 
vraie : une consonne géminée dans le taqjmit ne correspond pas nécessairement à une géminée en tachelhit $: \underline{\text { immi }} \rightarrow$ timmawiwi $\left(\mathrm{n}^{\circ}\right.$ 54), $\underline{\text { ddu }} \rightarrow$ tiddawiwi ( $\left.{ }^{\circ} 56\right)$ mais aussi bien ini $\rightarrow$ tinnawiwi $\left(n^{\circ} 49\right)$ et $\underline{s u} \rightarrow$ tissawiwi ( $\left.n^{\circ} 47\right)$. Il en va de même pour les consonnes radicales rédupliquées. Dans 3gugl $\rightarrow$ tiz3agljugl ( ${ }^{\circ}$ 20), la réduplication de $g$ dans la forme taqjmit n'est pas l'image de celle que présente la forme tachelhit : cette réduplication de la seconde radicale intervient en taqjmit systématiquement, que la consonne concernée soit simple ou rédupliquée en tachelhit. Le caractère géminé ou rédupliqué d'une $\mathrm{C}$ du taqjmit est donc totalement indépendant de la source tachelhit : il ne peut résulter que d'une contrainte de niveau gabaritique propre à ce langage secret.

Il convient ensuite de préciser la nature de ces éléments consonantiques $\mathrm{C}$ communs au tachelhit et au taqjmit. Le point concerne les consonnes de niveau morphologique, flexionnelles et dérivationnelles.

Les consonnes morphologiques flexionnelles du tachelhit ne sont pas conservées dans les formes taqjmit correspondantes. Aucune des consonnes de ce niveau dans les formes tachelhit de [1] ne se retrouve dans les formes correspondantes en taqjmit : ni le $t$ final de tahanut ( $\left.n^{\circ} 37\right)$, marqueur de féminin, ni le $t$ final de tnnit $\left(\mathrm{n}^{\mathrm{os}} 50,51,52\right)$, marqueur de $2 \mathrm{~s}$. Dans quelques cas, le $t$ initial des formes déguisées pourrait laisser penser qu'il reprend un $t$ initial, de nature morphologique (marqueur de personne), présent en tachelhit : ainsi dans tnnit $\rightarrow$ tinnawiwi $\left(\mathrm{n}^{\circ} 50\right)$ ou tigmmi $\rightarrow$ tiggamjumi $\left(\mathrm{n}^{\circ} 40\right)$. Mais toutes les formes en taqjmit débutent invariablement par $t$. Le $t$ initial du taqjmit n'est donc pas un élément récupéré dans la source, il n'est qu'un élément de brouillage préfixé : la forme tinnawiwi déguise aussi bien l'aoriste ini que l'accompli tnnit ( ${ }^{\text {os }} 49$ et 50).

Pour ce qui est des consonnes dérivationnelles, la situation est différente. On retrouve en effet dans les formes taqjmit des éléments consonantiques affixaux de niveau dérivationnel du tachelhit. C'est le cas du s- initial, morphème de causatif, et du m- initial, morphème de réciproque. Ainsi le s- de causatif de smun, ssry, ssknu ( $\mathrm{n}^{\text {os }} 8,14$ et 17$)$ et très probablement aussi de sgl, sawl et sadmr $\left(\mathrm{n}^{\text {os }}\right.$ 
$5,9$ et 22$)^{4}$ est conservé dans les formes déguisées (tissamnjumn, tissary jury, tissaknjukn, tissagljugl, tissawljuwl et tissadmrjumr respectivement). De la même façon le m- de réciproque de mmuddu ( $\left.\mathrm{n}^{\circ} 41\right)$ est conservé dans la forme taqjmit correspondante timmadjudi. Il est notoire que ce type de morphème consonantique dérivationnel est souvent traité comme du matériel radical. Idrissi 2001: 168-69 indique ainsi en tamazight l'existence d'une stratégie « d'incorporation » du matériel affixal dans la racine et la met en rapport avec la nécessité de satisfaire le gabarit de la formation. Les noms $\operatorname{ankkar}^{\complement} \mathrm{fu}$ (du verbe $\mathrm{krr}^{\mathrm{f}} \mathrm{f}$ être rejeté) et aqssajfu (du verbe qssf être près), par exemple, répondent à un gabarit commun -CCCVCCV, mais tandis que le second satisfait ce gabarit en insérant un $j$ entre la deuxième et la troisième radicales, le premier le fait en "incorporant" le $n$, qui est de niveau dérivationnel ( $n$ de réciproque $)^{5}$. L'incorporation des consonnes dérivationnelles dans le matériel radical en taqjmit semble motivée par le même type de contrainte gabaritique : cette incorporation affecte en effet (sauf sadmr, $c f$. infra) des verbes biconsonantiques et doit s'interpréter comme une stratégie parmi d'autres pour ramener des verbes biconsonantiques vers un modèle commun triconsonantique.

Il apparaît en effet que le taqjmit répond, de façon générale, à une contrainte fondamentale qui exige que toutes les formations comportent trois consonnes de statut radical, contrainte que nous dériverons de la structure du gabarit. Cette contrainte apparaît nettement dans le traitement des formations quadriconsonantiques du tachelhit.

Dans les quadriconsonantiques, on assiste en effet à un processus d'élimination d'un élément consonantique. La consonne éliminée, $m$ dans mbark

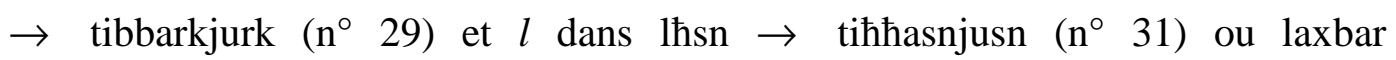
$\rightarrow$ tixxabrjubr ( $\left.\mathrm{n}^{\circ} 21\right)$, l'est probablement parce qu'elle est sentie comme de

\footnotetext{
${ }^{4}$ Contrairement aux autres formes citées, il n'y a pas pour ces trois verbes de forme de base (non causative) attestée. Pour sawl parle ! toutefois, le caractère dérivé est garanti par le nom awal parole.

${ }^{5}$ Pour une analyse alternative en termes de «stem-to-stem derivation » et « melodic transfer » à la suite de Hammond 1988, voir Dell \& Elmedlaoui 1992.
} 
nature affixale : dans ces mots, qui sont des emprunts à l'arabe, $m$ et $l$ sont en effet respectivement le morphème du participe et l'article défini. Mais dans le cas de brahim $\rightarrow$ tibbarhjurh et kltum $\rightarrow$ tikkatmjutm ( $n^{\text {os }} 30$ et 32), la consonne éliminée est une sonante qui n'est pas affixale, selon toute apparence - sans qu'on puisse, en l'occurrence, expliquer pourquoi la locutrice élimine la nasale $m$ dans le premier mais la liquide $l$ dans le second ${ }^{6}$. Cette stratégie peut être rapprochée de celle à laquelle on assiste dans la formation des pluriels nominaux de quinquilitères en arabe classique. Ceux-ci perdent automatiquement une consonne radicale dans la forme de pluriel e. g. safarzal (sg) > safaariz (pl) coing, barnaamaz (sg) > baraami3 (pl) programme, Sankabuut (sg) > Sanaakib (pl) araignée, ce qui est classiquement imputé au fait que le gabarit ne comporte que quatre positions C ( $c f$. McCarthy 1979 : 187, Moujib 1989 : 169-216, Kihm 2003 : 116).

En résumé, dans le cas où il y a plus de 3 consonnes dans l'input, ce sont les consonnes affixales qui sont prioritairement éliminées. Les consonnes flexionnelles le sont toujours, les consonnes dérivationnelles parfois.

Toutefois quatre formations quadriconsonantiques en [1], sadmr $\rightarrow$ tissadmrjumr, ffrtl $\rightarrow$ tiffartljutl, ggrml $\rightarrow$ tiggarmljuml et gg ${ }^{\mathrm{w}} \mathrm{nzr}$ $\rightarrow$ tigg ${ }^{\mathrm{w}}$ anzrjuzr ( $\left.\mathrm{n}^{\mathrm{os}} 22-25\right)$, gardent toutes leurs consonnes dans la forme déguisée alors qu'on s'attendrait à ce que leur nombre soit ramené à trois par élimination d'une consonne comme dans mbark ou laxbar. Le déguisement conduit ainsi pour ces quatre formes à un schème, $t i C_{1} C_{1} a \underline{C}_{2} C_{3} C_{4 j} u C_{3} C_{4}$, déviant par rapport aux schèmes listés en [2]. Mais on notera qu'un comportement particulier désigne clairement dans ces formes la consonne "surnuméraire" ( $d$ dans sadmr, $r$ dans ffrtl et ggrml, $n$ dans gg ${ }^{\mathrm{W}} \mathrm{nzr}$ ) : cette consonne n'est présente qu'une fois en taqjmit, c'est-à-dire qu'elle échappe à toute forme de réduplication. On

\footnotetext{
${ }^{6}$ Le cas de fatim $\left(n^{\circ} 43\right)$, en revanche, intrigue: on ne s'attend pas ici à l'élimination d'une consonne radicale puisque le mot est triconsonantique ; la raison pour laquelle la locutrice élimine le $m$ dans ce mot triconsonantique nous reste opaque. Au demeurant, on ne peut pas écarter absolument la possibilité d'une simple «erreur» de la locutrice. Confrontée à certaines « anomalies » comparables, Douchaïna 1998 : 207-208 semble bien, implicitement, envisager une certaine marge idiosyncrasique des locutrices.
} 
notera aussi que, si la nature de la consonne est variable (bien que les sonantes dominent), il s'agit toujours de $\mathrm{R}_{2}$. Le statut particulier de cette radicale et des sonantes qu'elle héberge généralement dans les formations quadriconsonantiques en afroasiatique est bien connu ( $c f$. entre autres Elmedlaoui 1994, Angoujard \& Denais 1989 : 106). Il est possible que ce statut particulier se manifeste, dans le cas qui nous occupe, au travers et de la conservation de la consonne et de son invisibilité au processus général de répétition qui sous-tend le taqjmit.

Quoi qu'il en soit, le traitement des formations quadriconsonantiques du tachelhit apparaît conflictuel, puisqu'on a dans certains cas élimination d'une radicale, et dans d'autres maintien de la totalité des radicales mais assorti d'une importante anomalie dans les processus de réduplication. Ce caractère conflictuel appuie fortement a contrario, nous semble-t-il, le caractère fondamentalement triconsonantique que nous attribuons au taqjmit : s'il n'était pas tel, en effet, on ne comprendrait pas ces flottements dans le déguisement en taqjmit des formations quadriconsonantiques du tachelhit.

En résumé, si on met à part les quatre formes quadriconsonantiques déviantes mentionnées ( $\left.\mathrm{n}^{\text {os }} 22-25\right)$ pour lesquelles nous n'avons pas d'analyse à proposer, il apparaît que, de l'information consonantique présente dans la forme tachelhit, on ne retrouve en taqjmit que :

a. l'information radicale [ou "incorporée"] ;

b. trois consonnes radicales maximalement

Les formes de tachelhit et celles de taqjmit partagent ainsi un seul objet : la racine consonantique ${ }^{7}$. C'est cette entité lexicale que le taqjmit manipule en définitive, dans son intégralité ou partiellement ( $c f$. 3b) et à l'exclusion de tout autre élément présent dans la forme tachelhit.

\footnotetext{
${ }^{7}$ Sur la notion de racine en berbère et en sémitique $c f$. entre autres Basset 1929 ; Galand 1988 ; Cohen 1988 ; McCarthy 1979 ; Diakonoff 1970 ; Prunet, Béland \& Idrissi 2000.
} 
Ce qui détermine ensuite la sélection de l'un ou l'autre des trois schèmes listés en [2] est clair : le schème adopté dépend directement et seulement du nombre d'éléments consonantiques radicaux disponibles ${ }^{8}$ :

[4]

\section{tachelhit}

a. trois consonnes radicales

b. deux consonnes radicales

c. une consonne radicale

$$
\Rightarrow \quad \text { taqjmit }
$$

$$
\begin{array}{ll}
\text { t i } \mathbf{C}_{1} \mathbf{C}_{1} \text { a } \mathbf{C}_{2} \mathbf{C}_{3} \text { j u } \mathbf{C}_{2} \mathbf{C}_{3} & c f .[1 \mathrm{a}] \\
\text { t i } \mathbf{C}_{1} \mathbf{C}_{1} \text { a } \mathbf{C}_{2} \mathbf{j} \text { u } \mathbf{C}_{2} \mathbf{i} & c f .[1 \mathrm{~b}] \\
\text { t i } \mathbf{C}_{1} \mathbf{C}_{1} \text { a w i w i } & c f .[1 \mathrm{c}]
\end{array}
$$

Ces trois schèmes appellent maintenant une double observation :

a. le matériel consonantique radical est systématiquement présent deux fois dans la forme déguisée

b. cette répétition emprunte deux voies :

1. la gémination, qui concerne seulement et toujours la première consonne radicale (désormais $\mathrm{R}_{1}$ );

2. la réduplication, qui concerne la deuxième et, le cas échéant, la troisième radicale (désormais $\mathrm{R}_{2}$ et $\mathrm{R}_{3}$ ).

Les parties - consonantiques et vocaliques - non héritées de la forme tachelhit observables dans les formes déguisées, quant à elles, peuvent, en première analyse, se décrire informellement comme résultant des opérations suivantes :

1. préfixation de $t i$;

2. insertion de $a$ après $\mathrm{R}_{1}$ géminée ;

3. insertion de $j u$ après $\mathrm{R}_{3}$ en [4a] et après $\mathrm{R}_{2}$ en [4b] ;

4. suffixation de $i$ en [4b] ;

5. suffixation de wiwi en [4c].

\footnotetext{
${ }^{8}$ Une forme contrevient à la généralisation $[4 \mathrm{~b}]: \mathrm{zr}^{\mathrm{S}} \rightarrow \operatorname{tizzar}^{\mathrm{q}}$ wiwi $\left(\mathrm{n}^{\mathrm{o}} 57\right)$. Bien que la formesource comporte deux radicales, c'est le schème [2c] sur lequel est construite sa forme déguisée et non le schème $[2 \mathrm{~b}]$ : tizzar ${ }^{\uparrow}$ wiwi et non ${ }^{*}$ tizzar $^{\Upsilon}$ juri. Nous n'avons pas d'explication à proposer pour cette anomalie, mais on notera tout de même que le cas offre de nettes similitudes avec celui des quadriconsonantiques "déviants" $\left(\mathrm{n}^{\mathrm{os}} 22-25\right)$ évoqués supra : 1) la consonne au comportement anormal est la seconde radicale, 2) il s'agit d'une sonante, 3) elle n'a pas d'image rédupliquée.
} 
Au terme de ce premier examen des données et à partir des généralisations qu'il a permises, une série de questions se posent. En particulier, on aimerait comprendre la distribution des trois opérations morphophonologiques à l'œuvre dans le déguisement taqjmit (gémination, réduplication et affixation) : pourquoi par exemple est-ce systématiquement $\mathrm{R}_{1}$ qui est géminée plutôt qu'une autre radicale tandis que $\mathrm{R}_{2}$ et $\mathrm{R}_{3}$ sont seulement susceptibles d'être rédupliquées?

Quant à la forme des affixations à droite de la partie $t_{i} C_{l} C_{l} a$ - qui est commune aux trois schèmes, à savoir $j u, j u \ldots i$ et wiwi en [4a], [4b] et [4c] respectivement, elle intrigue : il saute aux yeux en effet que les trois affixes, en dépit de leur différence, consistent et consistent seulement en deux "ingrédients" mélodiques, I et $\mathrm{U}^{9}$. Pourquoi cette affixation revêt-elle la triple forme que l'on observe ?

L'existence d'une partie gauche invariante et commune aux trois schèmes, opposée à cette partie droite variable, pourrait inciter, comme nous l'avons déjà évoqué brièvement, à analyser les formes du taqjmit en termes de "prosodic circumscription", dans la perspective de McCarthy \& Prince 1990. Il est vrai qu'on pourrait dans ce cadre rendre compte de la partie invariante. Mais l'un des postulats fondamentaux du modèle de McCarthy \& Prince est l'existence d'un lien direct entre l'input de la formation et son output, permettant le "transfert" de propriétés prosodiques précises du premier vers le second (dans l'un des exemples proposés par ces auteurs, les pluriels brisés de l'arabe classique, la longueur vocalique de la syllabe finale du singulier est ainsi transférée dans la forme du pluriel, e. g. sultaan sultan > pl. salaatịin, zundüb criquet $>$ pl. Zanaadib). Or dans les faits qui nous occupent, aucun lien prosodique de ce type ne peut, à notre connaissance, être posé entre les formes taqjmit et les formes tachelhit. Le simple examen des données présentées en [1] permet, nous semble-t-il, de conclure que

\footnotetext{
${ }^{9}$ La notation adoptée ici et par la suite fait référence à la "Théorie des Eléments" telle qu'elle est exposée dans Kaye, Lowenstamm \& Vergnaud 1985. Le lecteur est prié de se reporter à cet article pour le détail.
} 
les formes taqjmit n'héritent aucune propriété prosodique des formes correspondantes en tachelhit.

Au-delà, ce qui sous-tend l'analyse de McCarthy \& Prince est que les processus morphologiques ne sont pas performés sur une racine consonantique abstraite mais sur des formes concrètes. Or il n'apparaît pas que l'on puisse prédire lequel des trois schèmes sera activé au vu des caractéristiques de surface (prosodiques / syllabiques) des formes tachelhit. C'est seulement, comme dit en [4], le nombre des unités radicales disponibles qui le permet. Ce que les formes tachelhit et taqjmit ont en commun, c'est bien la racine et elle seule.

En outre, nous ne voyons pas comment l'approche de McCarthy \& Prince, essentiellement prosodique, pourrait permettre de rendre compte, dans la partie prosodiquement non circonscrite (droite), de l'existence des régularités segmentales précises que nous avons mentionnées précédemment : ni le fait que les unités segmentales qui apparaissent dans les affixes soient toujours et seulement $\mathrm{I}$ et $\mathrm{U}$, ni surtout les trois formes différentes que cette affixation revêt en surface ne nous paraissent pouvoir être capturées adéquatement dans ce cadre.

$\mathrm{Au}$ contraire, comme nous l'avons annoncé dans l'introduction, nous pensons que les questions que les faits observés soulèvent trouvent des réponses naturelles dans une perspective incluant la présence d'un gabarit construit. La section 3 sera consacrée à la définition de ce gabarit.

Avant cela, toutefois, nous rappellerons brièvement, dans la section 2 qui suit, quelques points importants concernant la notion même de gabarit et la morphologie dite "gabaritique" ainsi que le cadre syllabique dit « CVCV» dans lequel nous avons choisi d'opérer.

\section{Points théoriques}

\subsection{Gabarit et morphologie gabaritique}

Par gabarit, nous entendons une suite définie d'unités CV dotée d'une structure interne et d'une activité morphologique indépendante du niveau segmental. 
L'idée d'un rôle morphologique du gabarit a été formalisée initialement dans les travaux de McCarthy 1979 et 1981 sur l'arabe classique et en particulier son système verbal. McCarthy considère le gabarit ("CV-skeleton") comme un morphème, porteur de catégories grammaticales, consistant en une suite de positions $\mathrm{C}$ et $\mathrm{V}$ agencées de manière particulière - l'ensemble de ces "templates" dérivant néanmoins d'une formule gabaritique générale unique (McCarthy 1981 : 386-387). Les morphèmes radicaux (et éventuellement affixaux), ainsi que les morphèmes vocaliques (porteurs d'autres catégories grammaticales), tous situés sur des lignes autonomes, sont associés au gabarit selon des lois d'associations précises et universelles. Ainsi, pour la racine $\sqrt{ } \mathrm{ktb}$ écrire, par exemple, on dérive les formes verbales [6b] sur les gabarits [6a] :

[6]

a. b.

$\begin{array}{lll}\text { Forme I } & \text { C V C V C } & \text { k a t a b } \\ \text { Forme II } & \text { C V C C V C } & \text { kat tab } \\ \text { Forme III } & \text { C V V C V C } & \text { ka a t a b } \\ \text { Forme IV } & \text { C V C C V C } & \text { 2 a k t a b } \\ \text { Forme VII } & \text { C C V C V C } & \text { n kat a b }\end{array}$

Dans la mesure où les opérations morphologiques d'où résultent les divers schèmes ont des localisations récurrentes et précises, Guerssel \& Lowenstamm 1993 proposent pour le système verbal de l'arabe classique un gabarit construit, unique, de forme :

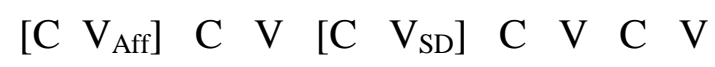

dans lequel $\left[\mathrm{CV}_{\mathrm{SD}}\right]$ est la "syllabe dérivationnelle", site de toutes les opérations morphologiques non concaténatives, et $\left[\mathrm{CV}_{\text {Aff }}\right]$ la syllabe affixale, site des opérations morphologiques de préfixation. La syllabe dérivationnelle comme la syllabe affixale sont des têtes morphologiques ; l'identification de leur position $\mathrm{C}$ ou de leur position $\mathrm{V}$ par du matériel segmental détermine le caractère dérivé de la forme. Dans ce cadre, les formes dérivées II, III, IV et VII de [6] ont respectivement les représentations $[8 \mathrm{a}],[8 \mathrm{~b}],[8 \mathrm{c}]$ et $[8 \mathrm{~d}]$ : 
[8] a.

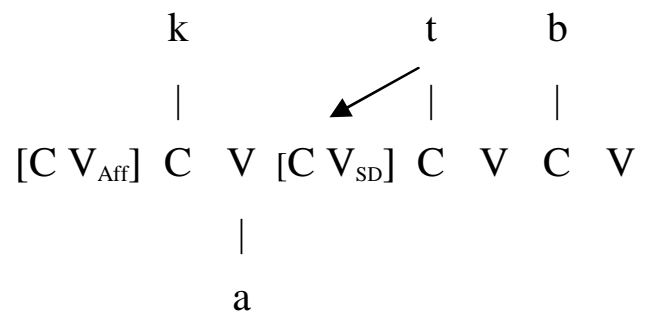

$[k a t t a b]$

c.

$?$

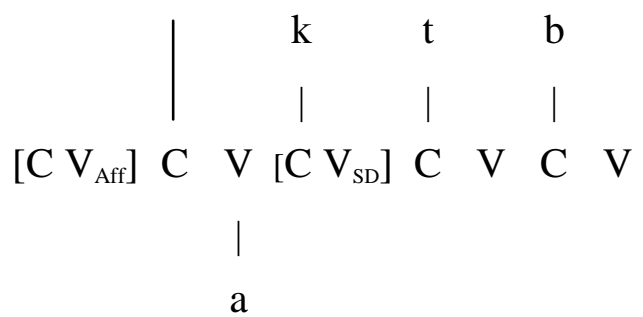

[?aktab] b.

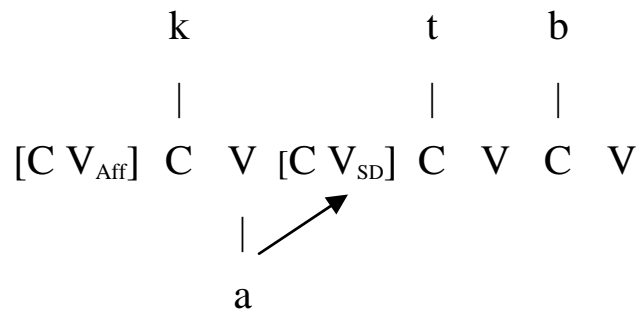

[kaatab]

d.

$\mathrm{n}$

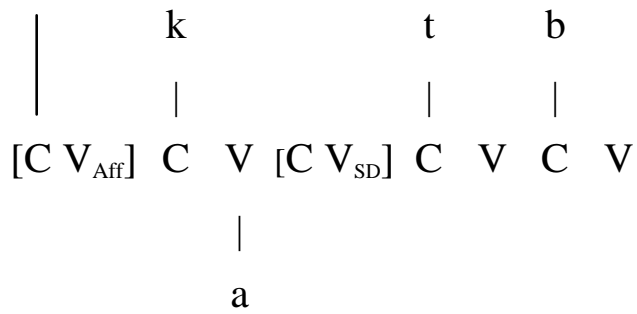

[nkatab]

La syllabe dérivationnelle est identifiée par $t$ en [8a], par $a$ en [8b] et par la racine en [8c]. En [8d], c'est la syllabe affixale qui est identifiée par l'affixe $n$. On dérive sur le même gabarit les autres formes, mutatis mutandis.

Des gabarits construits du même type ont été mis en évidence en afroasiatique par Aïm 2003 ; Bendjaballah 1999 ; Barillot 2002 ; Lahrouchi 2001, 2003 notamment.

\subsection{Le modèle « $C V C V »$}

Le cadre syllabique utilisé dans les représentations en [8] et dans lequel nous opérons dans ce qui suit est le modèle dit "CVCV" (Lowenstamm 1996a), lequel s'inscrit dans le cadre plus général de la Phonologie du Gouvernement. Ce modèle opère une réduction drastique des constituants syllabiques qui sont ramenés au nombre de deux, l'Attaque et le Noyau : la structure syllabique est une suite monotone d'Attaques et de Noyaux syllabiques simples (i.e. nonbranchants), notés $\mathrm{C}$ et $\mathrm{V}$. Les objets phonologiques traditionnels tels qu'une 
syllabe fermée, une consonne géminée et une voyelle longue ont respectivement dans ce cadre les représentations $[9 a],[9 b]$ et $[9 c]$ :

[9]

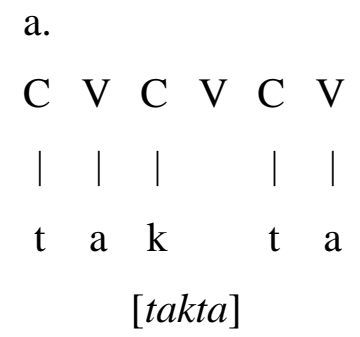

b.

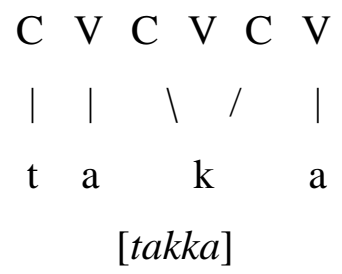

c.

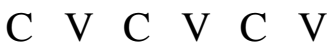

$\mathrm{t} \quad \mathrm{a} \quad \mathrm{k} \quad \mathrm{a}$ [taaka]

Dans ce modèle, les structures syllabiques arborescentes classiques sont réinterprétées en termes de relations latérales - Gouvernement et Licenciement que les Attaques et les Noyaux, et les segments qui leur sont associés, entretiennent entre eux. Ce sont ces relations latérales qui régulent le contenu des constituants et, en particulier, permettent à l'un ou à l'autre, dans des configurations précises, de rester ou non vide. Ainsi, comme représenté en [9a], la séquence consonantique interne [kt], traditionnellement analysée en une suite de coda + attaque, est réinterprétée dans le modèle CVCV comme une suite de deux attaques séparées par un noyau dont l'absence de contenu segmental est imputable à une forme particulière de Gouvernement, le "Gouvernement Propre", que le noyau suivant - phonétiquement exprimé - exerce sur lui. Le principe du Gouvernement propre trouve en grande partie sa justification en ce qu'il rend compte de la régularité des alternances voyelle/zéro qu'on observe dans des langues typologiquement aussi éloignées que le sont l'arabe maghrébin, le tchèque, l'allemand, le tangale...etc. ${ }^{10}$ Un exposé détaillé sur ces questions et sur le modèle CVCV d'une manière globale figure dans Scheer 2004.

\footnotetext{
${ }^{10}$ Nous n'ignorons pas que le berbère en général, et le tachelhit en particulier, soulèvent de sérieuses difficultés pour la Phonologie du Gouvernement et le modèle CVCV. Les langues berbères sont, en effet, riches en groupes consonantiques lourds. Dans certaines d'entre elles comme le kabyle, le tarifit ou le tamazight, une voyelle centrale brève (schwa) peut s'insérer phonotactiquement dans ces groupes consonantiques. Mais dans d'autres comme le tachelhit la chose est moins claire. Les linguistes sont d'ailleurs nettement divisés sur ce point : Dell \& Elmedlaoui 1985 ont proposé que les consonnes au même titre que les voyelles peuvent en tachelhit identifier le noyau syllabique et rejettent l'existence de schwas épenthétiques, d'autres (Coleman 1996, 2001, Louali \& Puech 2000, Puech \& Louali 1999) n'excluent pas la présence de voyelles réduites — au moins entre certaines consonnes, que Dell \& Elmedlaoui 2002: 135 d'ailleurs acceptent mais appellent des «voicoïdes transitionnels » lesquels sont définis comme
} 


\section{Le gabarit des bases triconsonantiques}

Comme dit en [5], le matériel consonantique radical est l'objet en taqjmit d'une répétition systématique. En sorte que le principe de base qui sous-tend la dérivation en taqjmit semble pouvoir, informellement, s'énoncer ainsi : "pour déguiser un mot, dites-le deux fois en un seul mot».

$\mathrm{Si}$ l'on considère le processus dans le cas d'un mot à radical triconsonantique, par exemple $\mathrm{k} \int \mathrm{m}$ entre ! ( $\mathrm{n}^{\circ} 3$ en [1]), la répétition semble concerner les consonnes de la racine et elles seules. En fait, nous pensons qu'il s'agit d'une illusion d'optique propre aux triconsonantiques (nous reviendrons plus bas en section 5 sur ce point en considérant les formations bi- et monoconsonantiques) et qu'en réalité, le principe de répétition en taqjmit s'énonce pour toute formation comme suit :

a. trois consonnes doivent être répétées

b. les trois consonnes répétées sont les consonnes (désormais $x, y, z$ ) associées aux positions $\mathrm{C}$ d'une portion du gabarit total, portion de forme : CVCVCV.

On notera que [10] implique que les positions $\mathrm{C}$ de cette portion du gabarit doivent être identifiées.

Par ailleurs nous avons constaté la présence, dans tous les cas, d'un préfixe dont l'élément consonantique est $t$ et d'un affixe dont l'élément consonantique est $I$ $(=[j])^{11}$.

On remarquera, avant de considérer les questions purement formelles que posent cette double affixation, que ces deux consonnes affixales correspondent,

« any voiced voicoid which is not an occurrence of one of the uncontroversial segments $i, a, u, y$, $w »)$. Bref, le point est complexe et très controversé, et en l'absence d'un consensus clair et assuré sur la présence ou non de schwas, le cadre théorique que nous avons choisi n'est pas plus disqualifié que les autres modèles théoriques concurrents. Aussi, sans nous dissimuler les problèmes qui demeurent, nous maintenons le cadre de représentation CVCV, et cela d'autant plus que, pour le problème précis qui nous occupe, cela n'a aucune incidence notable.

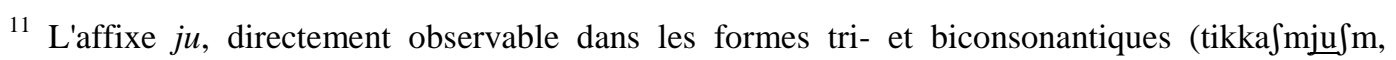
tigganjuni...) semble absent dans les formes mono-consonantiques : tiggawiwi. Mais cet affixe est bien présent sous-jacemment dans ces formes-là comme dans toutes les autres. Ce point sera clarifié dans la section 5 . 
dans la morphologie du tachelhit, à des marqueurs de genre : dans le système verbal, à l'accompli, à la $3^{\text {ème }}$ personne du singulier, $t$ marque le féminin et $I$ le masculin : tnkr elle s'est levée / inkr il s'est levé, tukr elle a volé / jukr il a volé, tiwi elle a emmené / jiwi il a emmené ${ }^{12}$. Or le taqjmit est un langage secret utilisé par un sous-ensemble sexuellement défini de locuteurs (des femmes, en l'occurrence). Il est ainsi tentant d'interpréter l'introduction simultanée, dans chaque formation, de deux affixes correspondant précisément aux deux marqueurs de la catégorie du genre comme une neutralisation de cette catégorie, neutralisation qui pourrait constituer la seconde « règle du jeu » du taqjmit ${ }^{13}$.

Quoi qu'il en soit, sur le plan formel, les deux affixes du taqjmit, $t$ et $I$ (ce dernier surfaçant dans le j de -ju- $c f$. [12] infra) occupent chacun une unité affixale CV située l'une à gauche et l'autre à droite de la partie CVCVCV définie en $[10 b]$.

La base sur laquelle est performée l'opération de répétition est donc de la forme représentée en [11], où le soulignement de la portion CVCVCV centrale indique, informellement, que les trois consonnes $x, y$ et $z$ associées aux positions $\mathrm{C}$ de cette portion doivent être répétées comme énoncé en [10] :

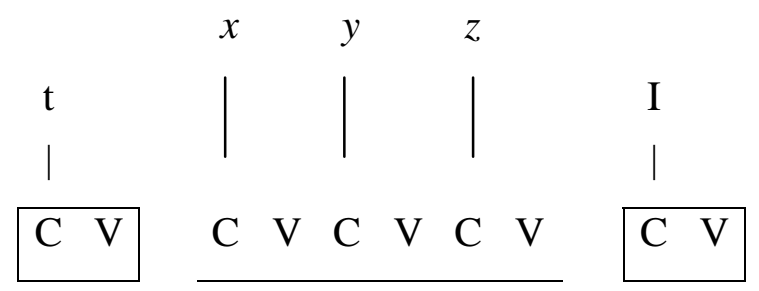

La question qui se pose alors est : pourquoi, étant donné cette structure de base, la contrainte de répétition amène-t-elle la gémination de $x$ mais la

\footnotetext{
${ }^{12}$ Dans les deux derniers verbes cités, la voyelle qui suit les marqueurs de genre $t$ - et $j$-appartient au radical et ne joue aucun rôle dans l'expression du genre. En taqjmit, le $i$ qui suit invariablement le $t$ comme le $u$ qui suit invariablement le $j$ relèvent de la vocalisation invariante des formes (voir section 4) et ne jouent aucun rôle dans le marquage du genre. Noter que le morphème $j$ se vocalise en $i$ devant un radical à initiale consonantique (ex. inkr $<\mathrm{j}$-nkr).

${ }^{13}$ Dans le tagnawt, autre langage secret féminin tachelhit décrit par Douchaïna 1998, on note une intervention différente mais portant aussi sur le marquage du genre : les formes du tagnawt sont uniformément préfixées par la particule aj- : tach. skr $\rightarrow$ tagn. ajssakrwakr fais! (Douchaïna 1998 : 198). Or celle-ci contient un marqueur masculin. Dans la morphologie verbale du tachelhit, en effet, cette particule résulte de la contraction d'un élément modal $a d$ et de $j$ - ou $i$-, lequel est comme il a été dit plus haut un marqueur de $3 \mathrm{~ms}$.
} 
réduplication de $y$ et $z$ ? Rien n'empêcherait a priori, en effet, que les consonnes soient répétées toutes les trois par réduplication à droite, comme figuré en [12] :

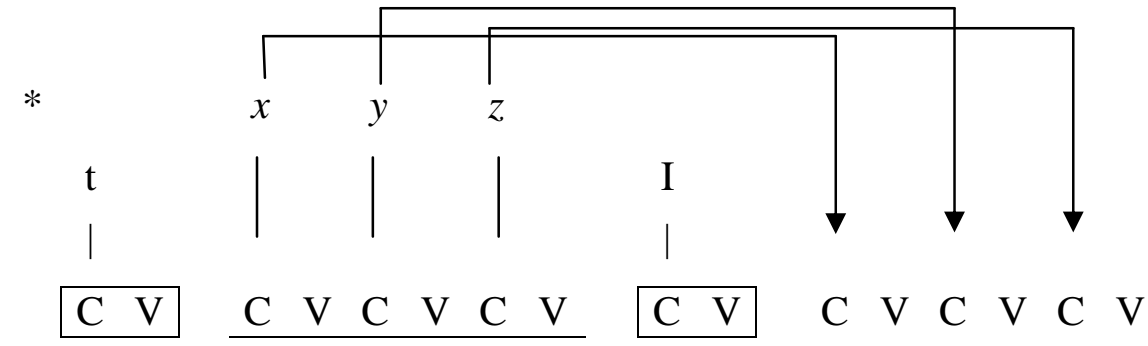

On dériverait ainsi, à partir de $\mathrm{k} \int \mathrm{m}$ par exemple, *tika $\int \mathrm{mjuk} \int \mathrm{m}$. Les contraintes de [10] seraient pleinement satisfaites. Mais ce n'est pas là ce qu'on

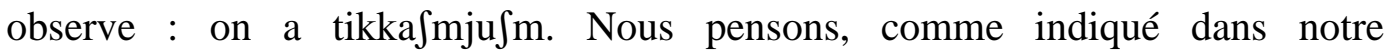
introduction, que la distribution des deux modes de répétition du matériel consonantique ne peut être capturée que comme une résultante de la structure du gabarit du taqjmit.

En négligeant pour l'instant la partie droite où sont associées les images des segments rédupliqués, nous proposons que toutes les formes taqjmit sont dérivées non sur le gabarit en [11] mais sur celui représenté ci-dessous en [13] :

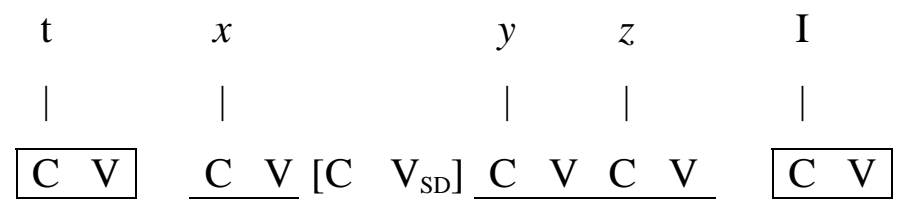

Comme on le voit, ce gabarit inclut crucialement un site dérivationnel $\left[\mathrm{CV}_{\mathrm{SD}}\right]$ à droite du premier $\mathrm{CV}$ de la portion $\underline{\mathrm{CVCVCV}}$. Nous affirmons que c'est la présence d'un tel site qui induit dans tous les cas la gémination de la consonne $x$ et d'elle seule. Dans la mesure où la forme de taqjmit est une forme dérivée, en effet, sa syllabe dérivationnelle doit être identifiée. Or elle ne peut l'être que par la consonne $x$. On pourrait imaginer que ce soit la consonne $y$ qui, se propageant sur la position $\mathrm{C}$ du site dérivationnel, assure cette identification. Dans ce cas, la consonne $x$ n'aurait aucun moyen de se répéter pour satisfaire la contrainte de répétition [10], sinon par réduplication à droite. On aurait, pour $\mathrm{k} \int \mathrm{m}$, quelque chose comme *tika $\iint m j u k m$ ou $*$ tika $\iint m j u m k$. Mais une telle réduplication 
reviendrait en fait à un croisement de lignes d'association, universellement proscrit, entre $x$ et $y$ : le processus de réduplication est une seule opération s'appliquant unitairement aux segments concernés; or, dans le cas envisagé, celle-ci concernerait $x$ et $z$, c'est-à-dire des segments séparés, sur la même ligne, par un autre segment $(y)$ non soumis au processus ${ }^{14}$. Seule la consonne $x$ peut ainsi satisfaire [11] en géminant sur la position $\mathrm{C}$ de la syllabe dérivationnelle ; au contraire, $y$ et $z$, elles, ne peuvent être répétées que par réduplication, à droite, comme figuré en [14] :

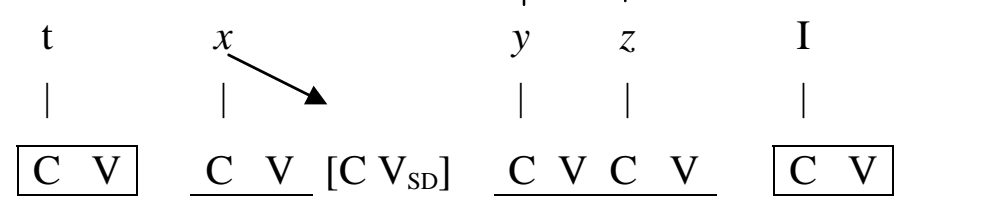

Si l'on considère maintenant plus attentivement la structure gabaritique que nous venons d'être conduits à supposer en [13] / [14] pour rendre compte de la forme que prend la répétition du matériel consonantique en taqjmit, il apparaît qu'elle correspond - si l'on oublie momentanément la syllabe affixale de droite où est ancré le $I$ - à [15] :

$$
\begin{array}{llllllllllll}
\hline \mathrm{C} & \mathrm{V} & \mathrm{C} & \mathrm{V} & {\left[\begin{array}{llllll}
\mathrm{C} & \mathrm{V}_{\mathrm{SD}}
\end{array}\right]} & \mathrm{C} & \mathrm{V} & \mathrm{C} & \mathrm{V}
\end{array}
$$

[15] correspond exactement au gabarit proposé par Guerssel \& Lowenstamm 1993 pour l'arabe classique ${ }^{15}$. Nous avons déjà eu l'occasion de dire que cette structure gabaritique n'est pas limitée à l'arabe classique mais se retrouve à l'identique dans un grand nombre de langues afroasiatiques, berbère inclus. Si notre analyse est correcte, le taqjmit ne serait ainsi qu'une instanciation de plus de cette architecture largement attestée en afroasiatique.

\footnotetext{
${ }^{14}$ En [12], il pourrait sembler que la réduplication de $y$ et $z$ "croise" également la ligne d'association de $I$. Mais ceci n'est qu'apparent : le segment $I$ du morphème affixal réside sur une ligne morphologique distincte de celle où résident $x, y$ et $z$.

${ }^{15}$ Dans la mesure où la syllabe affixale $\left[\mathrm{CV}_{\text {Aff }}\right]$ ne pourra jamais être activée en taqjmit étant donné que la syllabe dérivationnelle $\left[\mathrm{CV}_{\mathrm{SD}}\right]$ est, elle, toujours activée, nous faisons l'économie, dans notre représentation en [15], de cette syllabe affixale $\left[\mathrm{CV}_{\mathrm{Aff}}\right]$. Mais, sur le fond, ceci n'implique pas de différence par rapport au gabarit de Guerssel \& Lowenstamm représenté en [7].
} 
Tournons-nous maintenant vers la partie droite des formations triconsonantiques en taqjmit, c'est-à-dire la partie qui commence avec l'unité affixale $\mathrm{CV}$ porteuse du $I$. En surface, cette partie droite comporte à la suite de cette unité affixale deux consonnes qui sont les images rédupliquées de $y$ et $z$. Comment convient-il d'interpréter cette partie droite?

L'hypothèse la plus simple consiste à penser qu'une unité $\mathrm{CV}$ porteuse d'un I est insérée à droite du gabarit de base [15] et que lui fait suite l'espace nécessaire pour que les images rédupliquées de $y$ et $z$ puissent s'associer, à savoir deux unités CV. Mais si l'on adopte cette vision, il faut renoncer définitivement à comprendre pourquoi l'unité affixale porteuse du $I$ est située là où on l'observe plutôt qu'ailleurs - après le CVCV de réduplication, par exemple. Sa présence immédiatement à droite de [15] ne découlerait en effet de rien.

Nous pensons au contraire que, loin d'être arbitraire, la place de l'affixe porteur de $I$ procède du fait que la partie droite est une copie de la partie gauche. C'est-à-dire que nous proposons de voir, à la base de la morphologie du taqjmit, une opération de réduplication d'une base, en l'occurrence celle que nous avons définie en [15].

Dans ce cadre, l'unité $\mathrm{CV}$ affixale porteuse de $I$ se trouve là où elle se trouve parce qu'elle est l'image rédupliquée de la syllabe affixale initiale porteuse de $t$. Les syllabes affixales, identifiées l'une par $t$ et l'autre par $I$, se trouvent au début de chacun des deux membres de la formation : la partie gauche est la base, marquée pour le féminin, et la partie droite son image, marquée pour le masculin (parties dénommées désormais " $F$ " et " $M$ " respectivement). Entre les deux segments $t$ et $I$, il existe comme pour $x, y$ et $z$, une forme de copie, mais assortie dans ce cas d'une modification du contenu segmental déterminée par une inversion $(\mathrm{F} \rightarrow \mathrm{M})$ située au niveau morphologique : I est la copie morphologiquement inversée de $t$.

Quant aux opérations de répétition des consonnes $x, y$ et $z$, c'est sur ce gabarit "double" qu'elles sont effectuées, selon les modalités définies plus haut (gémination / réduplication). 
L'ensemble des opérations de réduplication du gabarit de base, de copie / inversion $t>I$ et de répétition du matériel segmental sont récapitulées en [16] :

[16]

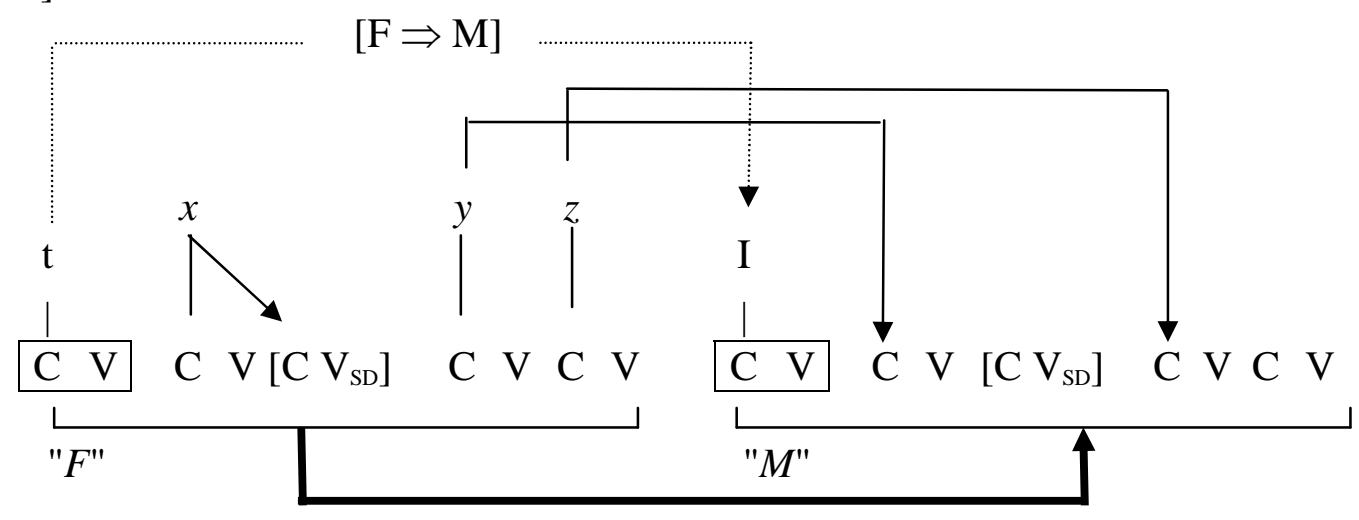

Précisons deux points concernant [16] :

a. dans le second membre " $M$ " de la formation, le $\left[\mathrm{CV}_{\mathrm{SD}}\right]$ dérivationnel n'est jamais identifié : le premier $\left[\mathrm{CV}_{\mathrm{SD}}\right]$ dérivationnel l'étant par la gémination de $x$, sa copie droite est fermée à toute opération morphologique ${ }^{16}$;

b. nous n'avons pas fait figurer en [16] la syllabe $\left[C V_{A f f}\right]$ que proposent Guerssel \& Lowenstamm 1993 dans le gabarit de l'arabe classique pour les raisons de clarté déjà indiquées dans la note 15. L'absence de cette syllabe dans notre représentation ne doit pas conduire à assimiler le CV qui accueille le tinitial (ni sa copie identifiée par l'élément I) au site $\left[C V_{A f f}\right]$ de Guerssel \& Lowenstamm 1993. Les sites morphologiques CV par lesquels débutent les deux portions " $F$ " et " $M$ " du gabarit en [16] se situent sur un palier («tier ») différent de celui de $\left[\mathrm{CV}_{\mathrm{SD}}\right]$ et $\left[C V_{A f f}\right]$. Pour le dire autrement, le parallèle n'est pas à faire entre n- de la forme VII perfective nkasar en arabe classique et t- / I- de taqjmit, mais bien entre ce dernier et ya- / ta- de la forme imperfective ya- / tankasir.

Ces points clarifiés, il reste une caractéristique importante dans notre représentation : en [16], le CV final de la partie droite " $M$ " n'est jamais identifié.

\footnotetext{
${ }^{16}$ Cette impossibilité renvoie à la question de l'architecture morpho-syntaxique des formes : deux syllabes dérivationnelles ne peuvent pas simultanément être activées dans une forme parce qu'une syllabe de ce type, au moins à un certain niveau dans l'architecture, est une tête (pour une discussion détaillée, $c f$. Aïm $2003: 36$ sqq.).
} 
$\mathrm{Au}$ vu de son "inutilité", on pourrait imaginer de faire tout simplement l'économie de ce $\mathrm{CV}$ final dans la représentation. Mais ceci conduirait à renoncer définitivement à un gabarit unique pour les formes du taqjmit. La même logique en effet qui imposerait de retrancher un $\mathrm{CV}$ du gabarit proposé à seule fin de "coller" à la forme de surface des triconsonantiques obligerait à en retrancher deux dans le cas des formations bi- et monoconsonantiques et donc à poser non plus un mais deux gabarits. Les triconsonantiques en effet comptent $8 \mathrm{CV}$ en surface mais les bi- et monoconsonantiques n'en comptent jamais que 7 :

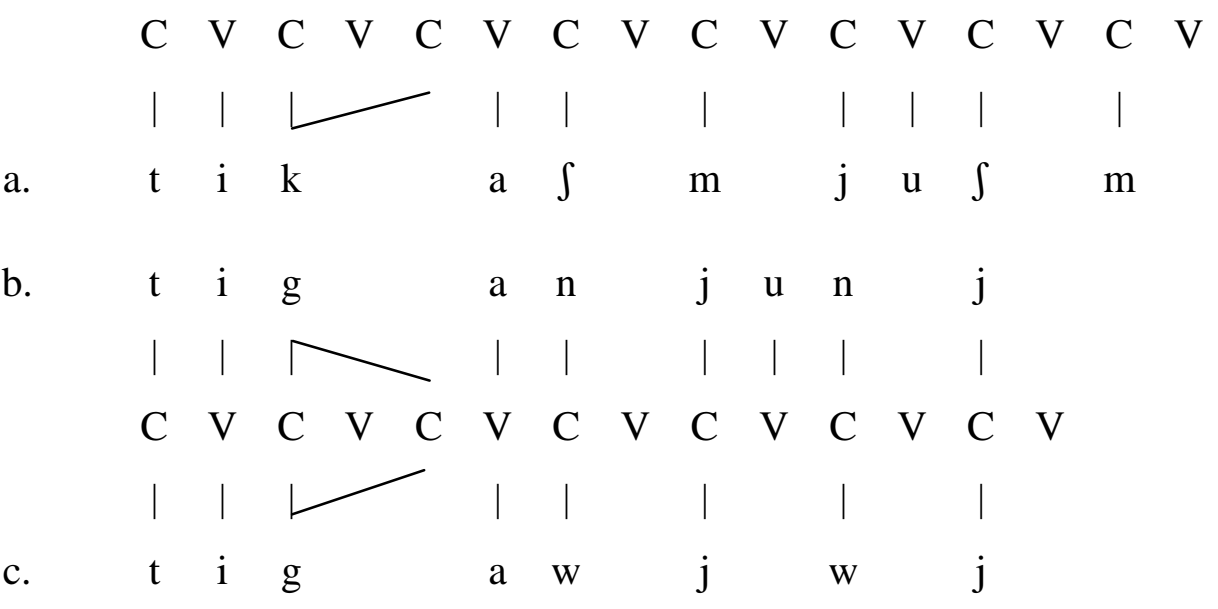

De façon plus préjudiciable encore, cela conduirait à renoncer à l'existence même d'un principe unique en taqjmit. Les événements prosodiques et segmentaux ne seraient plus justiciables d'une généralisation unique : la répétition concernerait seulement le niveau segmental. Le gabarit, lui, serait construit sur la seule base du nombre de segments à y accommoder.

Et au demeurant, quelle raison pourrait-on avoir de ne pas maintenir ce CV final ? On notera d'abord qu'il n'y a pas là de difficulté de principe : non identifiée segmentalement une unité CV tombe, simplement. En vérité, la seule raison d'écarter le CV final serait uniquement dictée par un souci de fidélité par rapport à la forme de surface. Mais un gabarit ne se déduit pas, mécaniquement, des faits de surface : bien que leurs formes de surface soient différentes, il sera légitime (et désirable) de proposer pour ar. class. kataba il a écrit et kaana il a été un gabarit unique. Un gabarit s'établit selon un raisonnement qui 1. prend en compte un ensemble de faits et 2. permet un gain de généralisation. 
Pour son adéquation aux faits observables et pour les généralisations désirables qu'il permet, nous maintenons donc le gabarit proposé en [16]. Et plutôt que de voir dans ce $\mathrm{CV}$ non identifié (ou ces $2 \mathrm{CV}$, pour les bi- et monoconsonantiques) une anomalie, nous proposons d'y voir au contraire un fait particulièrement significatif. Car rien ne serait plus simple que de satisfaire les positions "orphelines" : l'épenthèse ou la propagation d'un segment sont des stratégies possibles et, en pareil cas, attendues. De surcroît, elles sont parfaitement disponibles dans la langue, comme le prouve le tagnawt. Dans cet autre langage secret féminin en tachelhit déjà évoqué dans la note 13 , et qui présente avec le taqjmit d'importantes similitudes structurelles, l'épenthèse et la propagation sont massivement et systématiquement utilisées. Epenthèse par $j$ (qui surface en i) ou $t$ lorsque une radicale manque : sala $\rightarrow$ ajssalwalli s'occuper de, anf $\rightarrow$ ajttanfwanf éviter ; propagation (qui aboutit à une gémination) de la copie de la seconde radicale lorsque la troisième manque $:$ sala $\rightarrow$ ajssalwalli s'occuper de (Douchaïna 1998 : 200, 207). On pourrait imaginer de même qu'en taqjmit apparaisse un élément épenthétique, par exemple $t$ : *tikka $\int \mathrm{mju} \int \mathrm{m} t$, ou que $\mathrm{R}_{3}$ ou bien $\mathrm{R}_{2}$ soit à

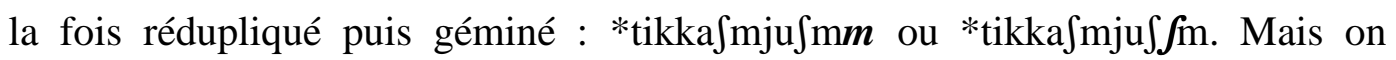
n'assiste jamais en taqjmit à aucun processus de ce type.

Le recours systématique à l'épenthèse et à la propagation répond à ce qui constitue, selon toute apparence, la contrainte fondamentale du tagnawt : une taille constante de toutes les formations, qu'elles soient tri-, bi- ou monoconsonantiques. De la même manière, mais a contrario, l'absence de tels procédés (et le caractère incomplet du gabarit qui en est la résultante) indique quelle est, en taqjmit, la "règle du jeu", règle que nous énonçons en [18] et désignerons sous le terme de principe de répétition stricte :

[18] tout élément de la base doit être répété, et une fois seulement

Un tel principe permet seul de capturer la totalité des observations que nous avons faites dans cette section. D'une part, en effet, il implique la répétition de la totalité du matériel consonantique associé à la base : 1. les 3 consonnes $\{x, y$, $z$ \} et 2. le $t$ affixal (modulo son "inversion" morphologique), ce qui est conforme à 
ce que l'on observe, et d'autre part il exclut tout élément épenthétique (un tel élément, non présent, par définition, dans la base, n'aurait de copie nulle part) et toute propagation de la copie d'un segment (qui serait alors présent trois fois), c'est-à-dire qu'il exclut ce qui, en effet, ne s'observe pas.

Notons enfin que l'incomplétude de la partie droite " $M$ " résulte certes du principe de répétition stricte [18], mais via la présence de la $\left[\mathrm{CV}_{\mathrm{SD}}\right]$ dérivationnelle de la base " $F$ " et de la nécessité de l'identifier. Si le gabarit n'incluait pas une syllabe de ce type, en effet, on aurait une réduplication pure et simple de type [12], et toutes les positions $\mathrm{C}$ du membre droit " $M$ " seraient alors identifiées. La syllabe dérivationnelle de " $F$ " n'est donc pas seulement responsable comme on l'a vu de la distribution des deux types d'opérations de répétition (gémination et réduplication), elle est également responsable de l'identification incomplète des positions $\mathrm{C}$ de la partie rédupliquée. Exprimé autrement, c'est le principe de répétition qui est responsable des aspects symétriques du taqjmit, et c'est la présence de la syllabe dérivationnelle de " $F$ " qui est responsable de tous les effets d'asymétrie qu'on y observe.

Dans ce cadre, la dérivation en taqjmit des mots à trois consonnes radicales, du type [4a] se fait simplement conformément à [16], les consonnes $x, y$ et $z$ correspondant dans ce cas aux trois consonnes radicales $\mathrm{R}_{1}, \mathrm{R}_{2}$ et $\mathrm{R}_{3}$, respectivement. Ainsi pour $\mathrm{k} \int \mathrm{m}$, on a tikka $\int \mathrm{mju} \int \mathrm{m}$ :

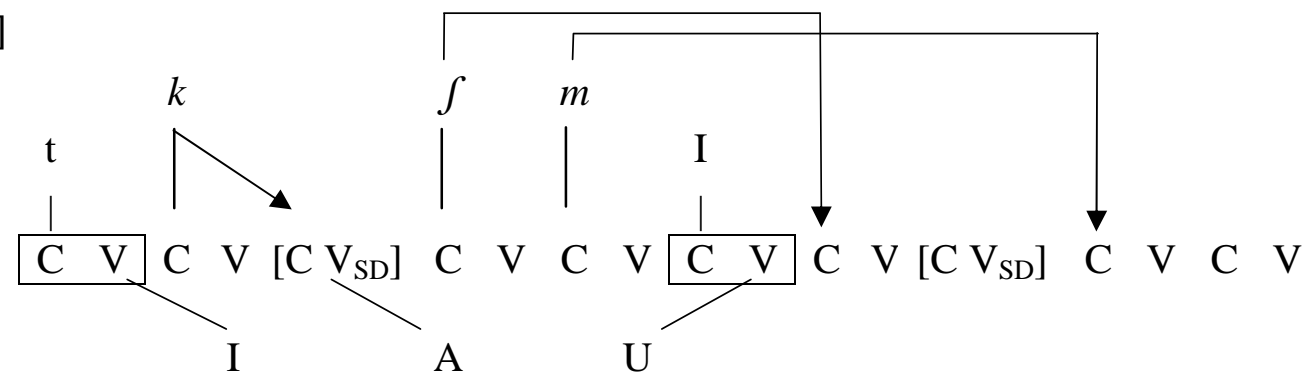

En section 5, nous considérerons les formations bi- [4b] et monoconsonantiques [4c] : l'analyse montrera que c'est le même gabarit unique [16] qui est responsable de la forme que revêtent en taqjmit ces formations. 
Mais avant cela, nous nous intéresserons dans la section 4 qui suit à la vocalisation des formes du taqjmit.

\section{L'origine apophonique de la vocalisation des formes du taqjmit}

Dans les formes du taqjmit issues de mots triconsonantiques de type [4a], la vocalisation consiste en une mélodie unique $i-a-u$ :

\begin{tabular}{|c|c|}
\hline$k \int m$ & tikkâsmjû $\int \mathrm{m}$ \\
\hline xadiz & 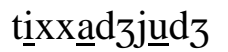 \\
\hline mbark & tibbbarkjü \\
\hline
\end{tabular}

Les mélodies des formes de taqjmit issues de bases bi- [4b] et mono- [4c] consonantiques, étant $i-a-u-i$ (gn $\rightarrow$ tigganjunini) et $i-a-i-i$ (ini $\rightarrow$ tinnnawiwwi) respectivement, s'écartent apparemment de cette mélodie invariante des triconsonantiques. En réalité, il ne s'agit là que d'un effet de surface : la mélodie vocalique sous-jacente est bien, dans ces formes bi- et monoconsonantiques la même que celle qui se montre à nu dans les triconsonantiques, à savoir $i-a-u$. L'analyse des bi- et monoconsonantiques dans la section 5 le montrera clairement. Si l'on veut bien admettre cette légère anticipation d'un résultat à venir, c'est donc à la généralisation suivante que l'on a affaire :

[20] la mélodie vocalique sous-jacente est dans toutes les formes du taqjmit :

$$
1 \quad A \quad U
$$

Quelle interprétation convient-il de donner à cette mélodie unique ? Il est frappant de constater que cette vocalisation mobilise la totalité des timbres vocaliques du tachelhit. Mais le point qui nous paraît crucial est l'ordre dans lequel la totalité de ces timbres apparaît : $i-a-u$, non $* u-i-a$, * $a-u-i$, etc.

Cet ordre ne nous paraît pas relever du hasard. Nous pensons que cette mélodie ainsi ordonnée doit être mise en rapport avec le mécanisme général soustendant tout processus apophonique selon la proposition de Ségéral 1995 et qui s'exprime sous la forme du « chemin apophonique » [21]:

$$
\phi \Rightarrow I \Rightarrow A \Rightarrow U \Rightarrow U
$$


L'existence d'une régularité apophonique reposant sur un ordonnancement invariant de timbres vocaliques a d'abord été mise en évidence par Guerssel \& Lowenstamm 1993 et 1996 pour les mutations apophoniques qu'on observe dans l'ensemble du système verbal de l'arabe classique et en particulier celles de la voyelle thématique dans la dérivation perfectif $\rightarrow$ imperfectif à la forme I : pf. labiss- $\rightarrow$ impf. -lbass- (I $\Rightarrow$ A), pf. katạb- $\rightarrow$ impf. -ktubb- $(A \Rightarrow U)$, etc. Une série de travaux récents a montré que la même régularité est à l'œuvre dans les systèmes apophoniques d'un grand nombre de langues sans parenté génétique ${ }^{17}$.

En berbère, cette régularité sous-tend les dérivations verbales et nominales (Bendjaballah 1995, 2001 et Idrissi 2000). Bendjaballah 2001 : 198 donne en kabyle l'exemple de la dérivation verbale aoriste $\rightarrow$ prétérit $:$ incliner mil $\rightarrow$ mal,

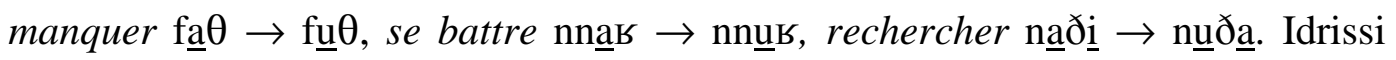
2000 : 122 présente le cas de la dérivation nominale singulier $\rightarrow$ pluriel en tamazight : joue madl $\rightarrow$ i-muddal, singe bayus $\rightarrow$ i-buyas, chevreau a-mkkartu $\rightarrow$

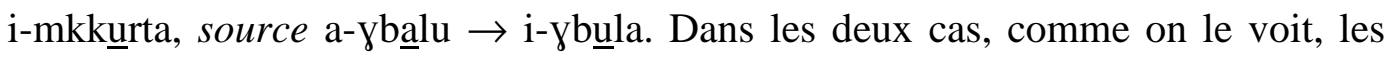
alternances vocaliques suivent le chemin apophonique [21]. En tachelhit-même, en l'absence d'une étude systématique des faits apophoniques, on ne peut affirmer que la régularité [21] sous-tend la totalité des dérivations apophoniques, mais bon nombre de ces dérivations au moins l'instancient clairement. C'est notamment le cas dans la dérivation verbale aoriste $\rightarrow$ prétérit : avoir ili $\rightarrow$ ila , vouloir iri $\rightarrow$ ira attraper amz $\rightarrow \mathrm{j}$-ümz, éviter anf $\rightarrow \mathrm{j}$-unf, etc. C'est également le cas dans la dérivation nominale singulier $\rightarrow$ pluriel $:$ forteresse a-gadir $\rightarrow$ i-gudar, langue ills

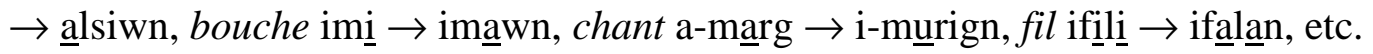

Il ne serait donc nullement étonnant de retrouver en taqjmit le recours à la même régularité.

Cela étant, nous ne sommes pas en taqjmit en présence de relations apophoniques entre deux formes dans un système grammatical. Nous avons

\footnotetext{
${ }^{17}$ Voir en particulier : Bendjaballah 1995, 1999 pour le berbère kabyle et pour le bédja, Idrissi pour le berbère tamazight, Ségéral \& Scheer 1996, 1998 pour l'allemand et le somali, Ségéral 1996 pour le ge'ez.
} 
affaire à la mélodie d'une forme et il n'y a évidemment pas de relation de "dérivation" entre les diverses parties de cette forme. En fait, nous sommes devant une exploitation du chemin apophonique d'un type différent de celle qui s'observe dans les systèmes morphologiques, nominaux ou verbaux. La formule apophonique peut, en effet, se concevoir, de manière plus générale, comme une organisation des primitives vocaliques $\{A, I, U\}$ (et de l'absence de contenu segmental $=\varnothing)$ selon un "gradient de marque". Des observations de niveaux divers (typologie des systèmes vocaliques, processus phonologiques tels que les harmonies ou l'épenthèse vocalique...) invitent à penser qu'il existe une hiérarchie de marque entre les voyelles et même entre les voyelles dans lesquelles les théories phonologiques reconnaissent généralement les primitives vocaliques, $i, a$ et $u$ : pour ne citer qu'un exemple, il est notoire que $i$ joue par excellence le rôle de "voyelle par défaut" (i.e. la moins marquée) ${ }^{18}$. Le chemin apophonique propose un encodage de la naturalité de cette hiérarchie de marque, conçue comme universelle, et disponible pour des exploitations linguistiques de natures diverses, non définies a priori. Or si l'on s'attend à ce qu'un tel gradient de marque soit évidemment exploité à des fins de marquage et de structuration de systèmes morphologiques, il n'est nullement exclu qu'il le soit à d'autres fins.

De même que la «réduplication» est un geste fondamental universellement disponible dans les langues utilisé, dans l'une, pour le marquage du nombre (e. g. somali sg. yár / pl. yaryár petit, sg. wanaagsán / pl. wanwanaagsán bon, Saeed 1993 : 194), dans une autre pour celui du fréquentatif (e. g. afar weele pleurer / weefàmweefe pleurer continuellement, Parker \& Hayward $1985: 251)$ et dans une autre encore à des fins expressives ( $c f$. fr. «ce

\footnotetext{
${ }^{18}$ La Théorie de la Sous-Spécification, entre autres, s'est efforcée de formaliser, au moins de façon paramétrique, ce corpus d'observations ( $c f$. par exemple, Stériade 1991). $i$ est la voyelle par défaut dans nombre de langues : Steriade 1991 : 132-140 rappelle, entre autres, les cas du yokuts, du yoruba (Pulleyblanck 1988) et de l'hébreu (où $i$ est la forme épenthétique en syllabe fermée initiale : Səmor mais Simrii), et on peut ajouter le tigrigna (Angoujard \& Denais 1989 : 136), l'akkadien (Ségéral $2000: 280$ ), ou encore la prothèse vocalique en latin vulgaire devant $* \#$ sC (schola > ischola) ou en arabe devant *\#CC (?inkasar-, Pistaqbal-), le traitement des emprunts avec *CC\# en somali (jaardi, inzi < angl. yard, inch, waqti < ar. waqt), etc. Le chemin apophonique prédit le statut de "voyelle par défaut" de i dans le premier pas du chemin : $\varnothing \Rightarrow \mathrm{i}$.
} 
n'est pas joli-joli ») etc., de même la formule apophonique est exploitée, certes, dans l'organisation de systèmes grammaticaux, comme on l'a évoqué ci-dessus, mais également comme un générateur de mélodies à des fins de vocalisation pour des formes dépourvues de vocalisation propre, lexicale ou grammaticalement motivée.

L'exemple le plus évident de cette dernière utilisation de l'apophonie étant les formations onomatopéiques / expressives. Il est frappant en effet, de constater que ces formations, comme l'avait déjà noté Grammont 1933 : 379, reprennent la même formule apophonique que celle qui a été mise en évidence ultérieurement par Guerssel \& Lowenstamm 1993 et 1996 dans le système verbal de l'arabe

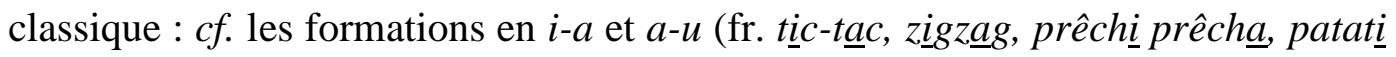
patata, zazou, cacou, badaboum, patapouf... ; all. flickflack, mischmasch,

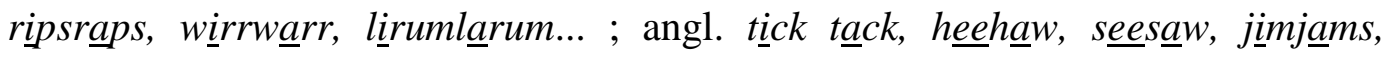

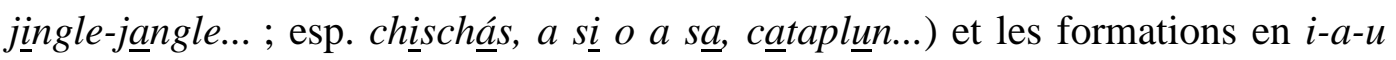

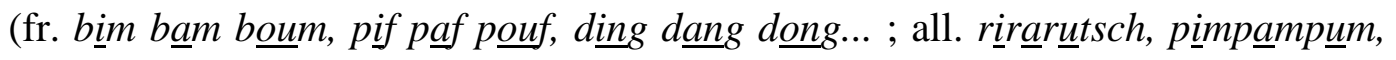

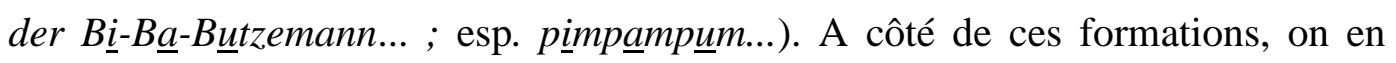
relève toutefois un certain nombre d'autres qui présentent un vocalisme $i$ - $o$, type tic-toc, flip-flop, ping-pong... On peut être tenté, en supposant un abaissement secondaire de la seconde voyelle arrondie, d'interpréter ce vocalisme en $i-u$, contraire à la régularité proposée. Mais cette interprétation est pour le moins aventurée : on a tic toc, pas *tic tuc et aucune de ces formations n'est en $i-u$. L'interprétation de la séquence $i$ - $o$ comme $i$ - $a$ pose certes inversement la question de l'arrondissement en $o$, mais reste néanmoins plus plausible — c'est d'ailleurs ainsi que Grammont (1933 : 379) analyse le timbre $o$ : "Quelquefois l'a est remplacé par un $o$ ouvert, de valeur à peu près équivalente". Et il est frappant que ces formations en $i$ - $o$ n'existent presque jamais seules : on a ces formes à côté de formes en $i$ - $a$ : on a tic toc et tic-tac, flip flop et flip flap, de bric et de broc à côté de bric-à-brac, cric-croc est attesté mais à côté de cric-crac, etc. Grammont (1933 : 380) mentionne encore l'existence de formations inverses par rapport au schéma proposé (e. g. lat. tux-tax) mais signale qu'elles sont "beaucoup plus rares" et se limitent aux formations à deux degrés (pas de $* u-a-i$ ). La séquence $i-a-u$ 
apparaît donc bien largement dominante dans les formations expressives à réduplication tandis que les séquences, a priori possibles, ${ }^{*} u-i, * i-u, * a-u-i$, *u-i$a . .$. , en sont généralement exclues. Or c'est par leur consonantisme onomatopéique d'une part et la présence de processus de réduplication d'autre part que ces formations sont « expressives » : leur mélodie n'est qu'un remplissage.

Nous pensons que la vocalisation du taqjmit relève de ce type d'exploitation de la séquence apophonique. Nous avons noté, en effet, dès notre premier examen des données que rien de la mélodie de la forme tachelhit n'était conservé dans le taqjmit. Celle qui apparaît en taqjmit, grammaticalement immotivée, n'est pas une manipulation, quelle qu'elle soit, de la mélodie de la source. Elle ne peut ainsi que résulter d'un "remplissage" mécanique. Et c'est, très naturellement, comme dans les formations onomatopéiques citées plus haut, le mécanisme apophonique qui le fournit.

Il est intéressant de noter pour clore cette section que le tagnawt, qui procède exactement au même type d'effacement de la mélodie de la source, recourt, lui, à une vocalisation tout aussi opaque, mais non modulée $(i$. $e$. n'exploitant pas le mécanisme apophonique). On a en effet partout $a$ : izwir précéder $\rightarrow$ ajzzawrwawr, rfufn peiner $\rightarrow$ ajrrafnwafn, zuzzr vanner $\rightarrow$ ajzzązzrwazzzr, etc (Douchaïna 1998). Dans les formations expressives, à côté des formations onomatopéiques à vocalisme modulé (= apophonique), on trouve aussi, significativement, des formations à vocalisme homogène, type fr. papa, coco, tutu, taratata, turlututu... Un vocalisme homogène et un vocalisme modulé selon le patron apophonique semblent les deux options possibles. Le tagnawt instantie la première option, le taqjmit la seconde : sa vocalisation est fondée sur la régularité apophonique [21].

\section{Les formations bi- et monoconsonantiques}

Dans le cas des bases triconsonantiques, nous avons vu en section 3 que les consonnes $x, y$ et $z$ correspondent aux trois radicales $\mathrm{R}_{1}, \mathrm{R}_{2}$ et $\mathrm{R}_{3}$. Dans celui 
des bases bi- et monoconsonantiques, le nombre de radicales est inférieur au nombre de consonnes qui doivent être rédupliquées. La question qui se pose alors est celle des stratégies utilisées en taqjmit pour satisfaire, malgré cette situation initiale défavorable, à la contrainte [10] qui exige que les trois consonnes $x, y, z$ soient rédupliquées.

Dans les biconsonantiques, la stratégie employée pour donner un contenu segmental à $z$ consiste à désassocier le $I$ de la position affixale et à le réassocier à gauche, les trois positions $x, y$ et $z$ étant identifiées respectivement par $\mathrm{R}_{1}, \mathrm{R}_{2}$ et $I$ :

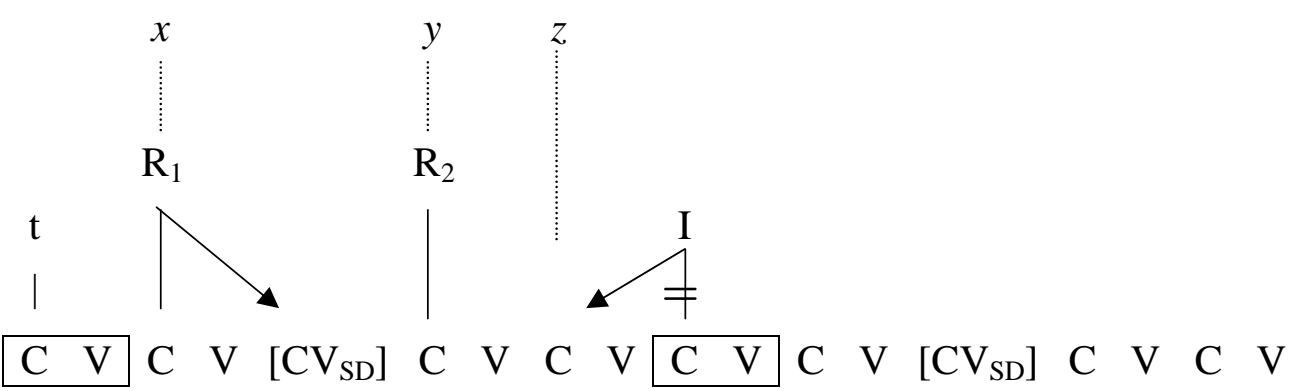

Si tel est le cas, alors $I$ doit, aux termes de [10], être rédupliqué. Or c'est bien ce qui se produit : le $i$ final qu'on observe dans toutes les formes taqjmit issues de bases biconsonantiques ( $c f$. [1b]) n'est que la forme vocalisée de la réduplication à droite du $I$. En tachelhit, en effet, un $I$ associé à une position $\mathrm{C}$ précédée et suivie d'un noyau vide est régulièrement vocalisé : ex. monter $\gamma^{\mathrm{w}} \mathrm{li}$ (aoriste) $v s$ aqq ${ }^{\mathrm{w}}$ lay (inaccompli), passer zri (aoriste) $v s$ zray (inaccompli). De même, en taqjmit le $j$ final dans /tigganjunj/ se vocalise en $i$ dans la forme de surface [tigganjuni] ${ }^{19}$. La représentation de la dérivation de tigganjuni $\leftarrow$ gn est donnée en [23] :

$$
\text { gn } \rightarrow \text { tigganjunj } \quad(\rightarrow[\text { tigganjuni }])
$$

${ }^{19}$ Le constat n'est pas propre au tachelhit : en tamazight, Guerssel (1986) a montué que si un [i] final peut représenter un /i/ ou un /j/, un /j/ final précédé d'une consonne surface invariablement en [i] (l'opposition tusyax «elle nous a portés » vs turiyax « elle nous a écrit » conduit à poser sousjacemment dans le premier un glide et dans le second une voyelle haute, mais à la forme $3 \mathrm{fs}$, on a [i] dans un cas comme dans l'autre : tusi « elle a porté » vs turi « elle a écrit »). 


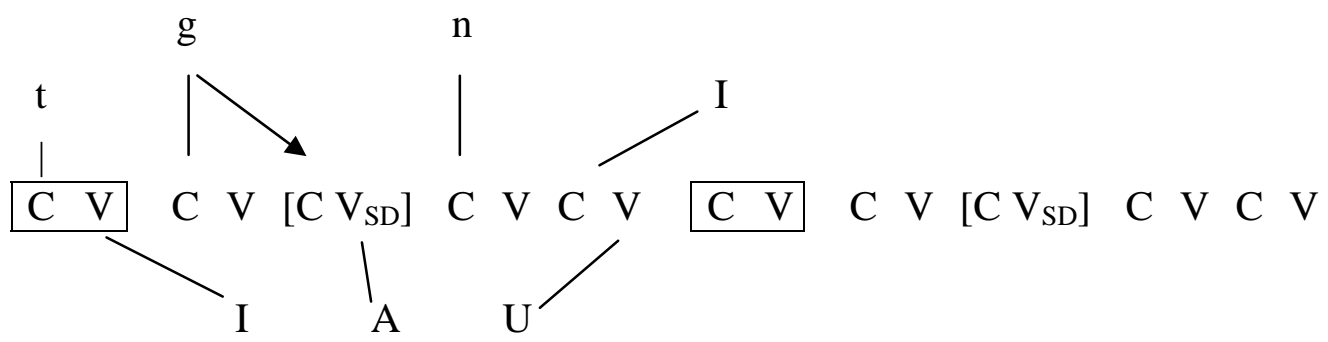

Le taqjmit montre, par le biais de ce type d'opérations, qu'en réalité ce ne sont pas les segments qui sont répétés, mais les positions gabaritiques. Peu importe l'origine affixale ou radicale des segments répétés : ce qui importe c'est qu'ils soient associés à l'intérieur de la base ${ }^{20}$.

On notera que la mélodie de surface $\left[\begin{array}{llll}i & a & u & i\end{array}\right]$ dans [tigganjuni] ne contrevient qu'en apparence, comme nous l'avions annoncé dans la section précédente, à la régularité apophonique : le $i$ final ne résulte que de la vocalisation phonotactique du $j$ et ne fait pas partie de la mélodie vocalique sous-jacente, laquelle est donc bien, conformément à [20] : I A U.

Dans les bases monoconsonantiques, la situation initiale est plus défavorable encore : le matériel consonantique radical se limite à $\mathrm{R}_{1}$ qui pourvoit au contenu segmental de $x$, mais ni $z$ ni $y$ n'ont de contenu segmental. La stratégie adoptée par le taqjmit dans cette situation reprend tout d'abord celle adoptée dans le cas des biconsonantiques : c'est le $I$ de la position affixale qui fournit son contenu segmental à $z$. Ensuite, il ne reste qu'une et une seule possibilité d'apporter un contenu segmental à la position $y:$ le $U$ de la mélodie apophonique. Toute solution utilisant le matériel consonantique aboutirait à transgresser le principe de répétition stricte : une consonne aurait à être présente trois fois. Et dans le matériel vocalique, seul $U$ est en mesure, par sa nature et sa position,

\footnotetext{
${ }^{20}$ Nous ne pouvons, dans les limites de cet article, traiter en détail de l'architecture morphosyntaxique des formes taqjmit. Nous préciserons brièvement, toutefois, les points suivants :

i. à l'intérieur du gabarit de base $\mathrm{CV}\left[\mathrm{CV}_{S D}\right] \mathrm{CV} \mathrm{CV}$, la tête morphologique est la syllabe dérivationnelle $\left[\mathrm{CV}_{S D}\right]$;

ii. à l'intérieur de la forme préfixée $\mathrm{CV}]+\mathrm{CV}\left[\mathrm{CV}_{S D}\right] \mathrm{CV} \mathrm{CV}$, la tête est $\mathrm{CV}$, identifiée par t- ;

iii. dans la forme rédupliquée $\mathrm{CV}+\mathrm{CV}\left[\mathrm{CV}_{S D}\right] \mathrm{CV} \mathrm{CV}-\mathrm{CV}+\mathrm{CV}\left[\mathrm{CV}_{S D}\right] \mathrm{CV} \mathrm{CV}$ i. e. la forme taqjmit (projection maximale), la syllabe préfixale initiale identifiée par t- reste la tête.

La désassociation du I de son site de départ, comme figuré en [23], n'affecte en rien cette architecture morphosyntaxique.
} 
d'identifier une position $\mathrm{C}$ : l'élément $A$ ne peut pas être associé à une position $\mathrm{C}^{21}$, et l'Elément $I$ de la mélodie, qui le pourrait par nature, ne le peut pas par position : son association à la position $\mathrm{C}$ orpheline croiserait la ligne d'association du $A$. Cette possibilité ultime d'identification de $y$ par le $U$ de la mélodie vocalique est bien celle qu'on observe en taqjmit : on a $w$ sur la position considérée, lequel est, comme attendu, rédupliqué. La forme taqjmit pour g "être" est :/tiggawjwj/, qui produit en surface [tiggawiwi] $]^{22}$ par suite de la vocalisation de $j$ déjà évoquée :

$$
\text { tiggawjwj } \quad(\rightarrow[\text { tiggawiwi }])
$$

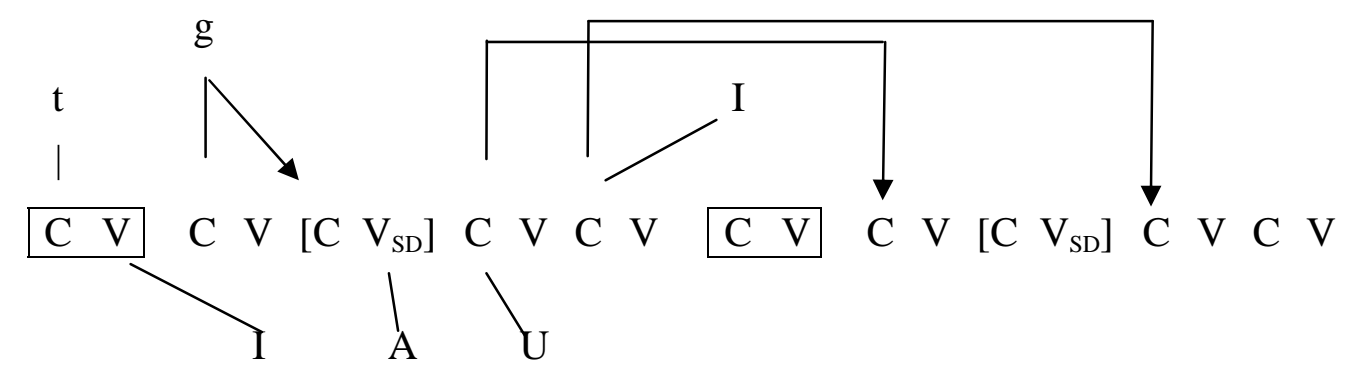

Et là encore, comme annoncé dans la section précédente, la mélodie est bien toujours I A $U$. L'absence en surface de $U$ ne résulte que de la "radicalisation" de cet élément, dûment présent dans la mélodie sous-jacente.

Pour clore cette section, on notera que le recours au I affixal pour pallier l'absence de $R_{3}$ et au $U$ de la mélodie pour pallier l'absence de $R_{2}$ dispense le taqjmit d'identifier les positions $y$ et $z$ orphelines par voie d'épenthèse. En tagnawt au contraire, comme on l'a vu, c'est par épenthèse que les mono- et biconsonantiques voient le matériel segmental de leur base complété (type anf $\rightarrow$ ajttanfwanf éviter). Mais le lecteur se souvient aussi que la mélodie du tagnawt est uniformément en $a$ ( $c f$. section 4 fin), c'est-à-dire d'une nature telle qu'elle ne peut pas être exploitée pour identifier des positions consonantiques qui doivent l'être.

\footnotetext{
${ }^{21}$ Prunet 1996 et 1998 propose qu'un élément A est en certains cas associé à des positions C. Pour Lowenstamm 1996b : 130-131, cet élément A est certes la trace vocalique d'une consonne gutturale disparue, mais il demeure associé à des positions V (a long). Même vision, semble-t-il, dans Petros Banksira 1997 : 31. Nous nous en tenons à cette dernière interprétation.

${ }^{22}$ Une autre possibilité est logiquement envisageable : I affixal et U de la mélodie pourraient, sans inconvénient, identifier dans le sens contraire les deux positions $C$ concernées, auquel cas on aurait *tiggajuju. Nous avouons n'avoir pas de raison à produire pour justifier le "choix" que fait ici la langue.
} 


\section{Conclusion}

Le taqjmit, langage secret féminin en berbère tachelhit, apparaît comme reposant sur une activité fondamentalement gabaritique: la distribution des différentes opérations morphologiques observables dans les formes de ce langage (gémination, réduplication et affixation) s'analyse comme déterminée par l'existence d'un gabarit construit qui consiste en la réduplication pure et simple d'un gabarit de base incluant un site morphologique vide de forme CV. C'est ce site qui implique en particulier la gémination systématique de la première consonne radicale dans les formes déguisées.

Sur le plan segmental, si la répétition, qui est le principe de base soustendant la dérivation en taqjmit, ne concerne dans le cas des mots triconsonantiques que les éléments de la racine et seulement ceux-là, nous avons montré que, dans le cas des bi- et monoconsonantiques, ce sont des éléments d'origine affixale ou mélodique qui viennent compléter le matériel segmental soumis à répétition. Le marqueur de genre qui est préfixé à toutes les formes en taqjmit n'échappe pas à la contrainte de répétition, mais la répétition se double dans son cas d'une inversion qui amène la présence simultanée, dans la forme, des marqueurs féminin et masculin. L'analyse des formes dans ce cadre permet de préciser la nature exacte de la contrainte de répétition à l'œuvre dans le taqjmit : tous les éléments de la forme de base (gabarit, matériels radical et affixal) doivent être répétés mais présents seulement deux fois dans la forme déguisée.

Quant à la vocalisation invariante des formes du taqjmit, elle s'analyse comme résultant de l'exploitation sans motivation grammaticale d'un mécanisme apophonique actif dans la langue mais présent largement au-delà du berbère.

Sur un plan plus général, les faits du taqjmit plaident clairement pour une morphologie de type root-and-template, dans laquelle 1. la racine consonantique est un morphème à part entière, auquel les locuteurs (-trices) ont accès : la forme taqjmit n'est pas dérivée directement à partir de la forme tachelhit, sa dérivation transite crucialement par l'extraction de la racine consonantique lexicale, dont les éléments présents en taqjmit sont les seuls présents aussi en tachelhit, 2. le gabarit 
exige un nombre fixe de segments consonantiques — dans le cas du taqjmit, trois, comme le montrent les stratégies observées lorsque le matériel segmental fourni par le lexique est inférieur (ou supérieur) à trois éléments consonantiques.

\section{Références}

Aïm, Emmanuel (2003). Questions de phonologie et de morphologie sémitiques. Thèse de Doctorat, Université Paris 7.

Angoujard, Jean-Pierre \& Michel Denais (1989). Le pluriel brisé en tigrigna. Langues Orientales Anciennes Philologie et Linguistique 2 : 99-148.

Bagemihl, Bruce (1987). Tigrinya Speech Disguise and Constraints on Spreading Rules. In Proceedings of WCCFL 6, M. Crowhurst (ed.) : 1-15. Stanford : SLA.

Bagemihl, Bruce (1988). Alternate Phonologies and Morphologies. University of British Columbia.

Bagemihl, Bruce (1989). The Crossing Constraint and "backwards languages", NLLT 7 : 481-549.

Bagemihl, Bruce (1991). Language Games and related areas. In The Handbook of Phonological Theory, J. A. Goldsmith (ed.) : 797-712. Cambridge MA / Oxford : Blackwell.

Barillot, Xavier (2002). Morphophonologie gabaritique et information consonantique en somali et dans les langues est-couchitiques. Thèse de Doctorat, Université Paris 7.

Basset, André (1929). La langue berbère. Morphologie. Le verbe - étude de thèmes. Edition 2004. Paris : L'Harmattan.

Bendjaballah, Sabrina (1995). Aspects du système verbal du berbère (kabyle). Mémoire de DEA, Université Paris 7.

Bendjaballah, Sabrina (1999). Trois figures de la structure interne des gabarits : activité morphologique du niveau squelettal des représentations phonologiques en berbère, somali et bedja. Thèse de Doctorat, Université Paris 7.

Bendjaballah, Sabrina (2001). The Negative Preterit in Kabyle Berber. Folia Linguistica 34,3-4 : 185-223. Berlin : Mouton de Gruyter.

Berjaoui, Nasser (1994). Preliminary notes on a secret language of «telba » in Morocco. In Actes des premières journées internationales de dialectologie arabe de Paris, D. Caubet \& M. Vanhove (eds) : 511-517. 
Boukous, Ahmed (1987). Phonotactique et domaines prosodiques en berbère. Thèse de Doctorat d'Etat, Université Paris 8.

Cohen, David (1988). "Le chamito-sémitique". In Les langues dans le monde ancien et moderne : langues chamito-sémitiques, J. Perrot \& D. Cohen (eds) III : 9-30. Paris : CNRS.

Coleman, John (1996). Declarative Syllabification in Berber Tashlhiyt. In Current Trends in Phonology : Models and Methods (2 vol.), J. Durand \& B. Laks (eds) : I-175-216. Salford / Manchester : ESRI.

Coleman, John (2001). The Phonetics and Phonology of Tashlhiyt Berber Syllabic Consonants. Transactions of the philological Society 99 : 29-64.

Dell, François \& Mohamed Elmedlaoui (1985). Syllabic Consonants and Syllabification in Imdlawn Tashlhiyt Berber. Journal of African Languages and Linguistics 7 : 105130.

Dell, François \& Mohamed Elmedlaoui (1992). Quantitative transfer in the nonconcatenative morphology of Imdlawn Tashlhiyt Berber. Journal of Afroasiatic Languages $3: 89-125$.

Dell, François \& Mohamed Elmedlaoui (2002). Syllables in Tashlhiyt Berber and in Moroccan Arabic. Dordrecht : Kluwer Academic Publishers.

Diakonov, Igor Mikhailovich (1970). Problems of root structure in Proto-Semitic. Archiv Orientalni 38 : 453-480.

Diakonov, Igor Mikhailovich (1988). Semitico-Hamitic languages. Afrasian Languages. Moscou.

Douchaïna, Rqia (1998). La morphologie du verbe en tagnawt. Etudes et Documents Berbères, 15-16 : 197-209.

Elmedlaoui, Mohamed (1994). Extension de la racine chamito-sémitique. Linguistique Africaine 12 : 93-118.

Galand, Lionel (1988). Le berbère. In Les langues dans le monde ancien et moderne : langues chamito-sémitiques, J. Perrot \& D. Cohen (eds) III : 207-242. Paris : CNRS.

Grammont, Maurice (1933). Traité de phonétique. Paris : Delagrave.

Guerssel, Mohand (1986). Glides in Berber and Syllabicity. Linguistic Analyis 17, 1 : 112.

Guerssel, Mohand \& Jean Lowenstamm (1993). Classical Arabic Apophony [ms]. Montréal (UQAM) \& Paris (Université Paris 7). 
Guerssel, Mohand \& Jean Lowenstamm (1996). Ablaut in Classical Arabic Measure I Active Verbal Forms. In Studies in Afroasiatic Grammar. Papers from the Second Conference on Afroasiatic Languages : Sophia-Antipolis, 1994. J. Lecarme, U. Schlonsky \& J. Lowenstamm (eds) : 123-134. The Hague : Holland Academic Graphics.

Hammond, Michael (1988). Templatic transfer in Arabic broken plurals. Natural Language and Linguistic Theory $6: 247-270$.

Hombert, Jean-Marie (1973). Speaking Backwords in Bakwiri. Studies in African Linguistics 4 : 227-258.

Hombert, Jean-Marie (1986). Word Games. Some Implications for Analysis of Tone and other Phonological Constructs. In Experimental Phonology, J. J. Ohala \& J. J. Jaeger (eds) : 175-186. Orlando : Academic Press.

Idrissi, Ali (1997). Plural formation in Arabic. Perspectives on Arabic Linguistics X. M. Eid \& R. R. Ratcliffe (eds) : 123-145. Amsterdam : John Benjamins.

Idrissi, Ali (2000). On Berber plurals. Research in Afroasiatic Grammar. J. Lecarme, J. Lowenstamm \& U. Shlonsky (eds) : 101-124. Amsterdam : John Benjamins.

Idrissi, Ali (2001). Towards a root-and-template approach to shape-invariant morphology. Thèse de Doctorat, UQAM.

Kaye, Jonathan (1990). "Coda" Licensing. Phonology Yearbook 7 : 301-330.

Kaye, Jonathan, Jean Lowenstamm \& Jean-Roger Vergnaud (1985). The Internal Structure of Phonological Elements : a Theory of Charm and Government. Phonology Yearbook 2 : 305-328.

Kaye, Jonathan, Jean Lowenstamm \& Jean-Roger Vergnaud (1990). Constituent Structure and Government in Phonology. Phonology Yearbook 7, 2 : 193-231.

Kihm, Alain (2003). Les pluriels internes de l'arabe: système et conséquences pour l'architecture de la grammaire. Recherches Linguistiques de Vincennes 32 : 109-155.

Lahrouchi, Mohamed (2001), Aspects morpho-phonologiques de la dérivation verbale en berbère (parler chleuh d'Agadir). Contribution à l'étude des gabarits, Thèse de Doctorat, Université Paris 7.

Lahrouchi, Mohamed (2003). Manifestations gabaritiques dans la morphologie verbale $\mathrm{du}$ berbère tachelhit. Recherches Linguistiques de Vincennes 32 : 61-82.

Louali, Naïma \& Gilbert Puech (2000). Etude sur l'implémentation du schwa pour quatre locuteurs berbères de tachelhit. Papier présenté aux XXIII ${ }^{\text {èmes }}$ Journées d'Etude sur la Parole : 19-23 juin 2000, Aussois. 
Lowenstamm, Jean (1991). Vocalic length and centralization in two branches of Semitic. In Semitic Studies in Honor of Wolf Leslau on the Occasion of his $85^{\text {th }}$ Birthday (2 vol.), A. S. Kaye (ed.) : II-949-965. Wiesbaden : Otto Harrassowitz.

Lowenstamm, Jean (1996a). CV as the only Syllable Type. In Current Trends in Phonology : Models and Methods (2 vol.), J. Durand \& B. Laks (eds) : II.419-441. Salford / Manchester : ESRI.

Lowenstamm, Jean (1996b). Five puzzling Chaha verbs : an exercise in practical morphophonemics. In Essays on Gurage language and culture, Grover Hudson (ed.) : 123-132. Wiesbaden : Harrassowitz.

McCarthy, John (1979). Formal Problems in Semitic Phonology and Morphology. Thèse de Ph.D., MIT.

McCarthy, John (1981). A Prosodic Theory of Nonconcatenative Morphology. Linguistic Inquiry $12: 373-418$.

McCarthy, John (1985). Speech Disguise and Phonological Representation in Amharic. In Advances in Nonlinear Phonology, H. van der Hulst \& N. Smith (eds) : 305-312. Dordrecht : Foris.

McCarthy, John (1991). L'infixation réduplicative dans les langages secrets. Langages $101: 11-29$.

McCarthy, John \& Alan Prince (1990). Foot and word in prosodic morphology : The Arabic broken plurals. Natural Language and Linguistic Theory 8 : 209-283.

Moujib, D. Ilham (1989). La formation des pluriels brisés issus des quinquilitères et des sextilitères en arabe. Languages and Linguistics 2: Issues in Arabic Morphology: Empirical and Theoretical Perspectives, A. Chekayeri \& G. Bohas (eds) : 93-145.

Parker, Enid M. \& Richard J. Hayward (1985). An Afar-English-French Dictionary (with grammatical notes in English). London : SOAS.

Petros Banksira, Degif (1997). The Sound System of Chaha. Thèse de Doctorat, UQAM.

Plénat, Marc (1984). Toto, Fanfa, Totor et même Guiguitte sont des anars. In Forme sonore du langage, F. Dell, D. Hirst \& J.-R. Vergnaud (eds) : 161-181. Paris : Hermann.

Plénat, Marc (1985). Morphologie du largonji des Loucherbems. Langages 78 : 73-122.

Prunet, Jean-François (1996). Guttural vowels. In Essays on Gurage language and culture, Grover Hudson (ed.) : 175-203. Wiesbaden : Harrassowitz.

Prunet, Jean-François (1998). When vowels function like consonants. In Phonological 
Studies 1, The Phonological Society of Japan (ed.) : 219-226. Tokyo : Kaitakusha.

Prunet, Jean-François, René Beland \& Ali Idrissi (2000). The mental representation of Semitic words. Linguistic Inquiry 31, 4 : 609-648.

Puech, Gilbert \& Naïma Louali (1999). Syllabification in Berber : the Case of Tashlhiyt. In Proceedings of the 14th International Congress of Phonetic Sciences (1-7 august 1999) : 747-750, San Francisco.

Pulleyblank, Douglas (1988). Vocalic underspecification in Yoruba. Linguistic Inquiry $19: 233-270$.

Saeed, John Ibrahim (1993). Somali reference Grammar. Kensington (Maryland) : Dunwoody Press.

Scheer, Tobias (2004). A lateral theory of phonology: what is CVCV, and why should it be. Berlin / New York : Mouton de Gruyter.

Ségéral, Philippe (1995). Une théorie généralisée de l'apophonie. Thèse de Doctorat, Université Paris 7.

Ségéral, Philippe (1996). L'apophonie en ge'ez. In Studies in Afroasiatic Grammar, (J. Lecarme, J. Lowenstamm \& U. Shlonsky (eds) : 360-391. The Hague : Holland Academic Graphics.

Ségéral, Philippe (2000). Théorie de l'apophonie et organisation des schèmes en sémitique. In Research in Afroasiatic Grammar II, J. Lecarme, J. Lowenstamm \& U. Schlonsky (eds) : 263-299. Amsterdam / Philadelphia : John Benjamins.

Ségéral, Philippe \& Tobias Scheer (1998). A generalized theory of Ablaut : the case of modern German strong verbs. In Models of Inflection, R. Fabri, A. Ortmann \& T. Parodi (eds) : 28-59. Tübingen : Niemeyer.

Steriade, Donca (1991). Underspecification and Markedness. In The Handbook of Phonological Theory, J. Goldsmith (ed.) : 114-174. Oxford / Cambridge MA : Blackwell.

Youssi, Ahmed (1977). Les parlers secrets au Maroc. La linguistique 13 : 135-143. 